THE ASTROPHYSICAL JOURNAL, 453:464-479, 1995 November 1

(5) 1995. The American Astronomical Society. All rights reserved. Printed in U.S.A.

\title{
SIMULTANEOUS EXTREME-ULTRAVIOLET EXPLORER AND OPTICAL OBSERVATIONS OF AD LEONIS: EVIDENCE FOR LARGE CORONAL LOOPS AND THE NEUPERT EFFECT IN STELLAR FLARES
}

\author{
SUZANNE L. HAWLEY ${ }^{1}$ \\ Department of Physics and Astronomy, Michigan State University, East Lansing, MI 48824 \\ GEORGE H. FISHER \\ Space Sciences Laboratory, University of California, Berkeley, CA 94720 \\ THEODORE SIMON \\ Institute for Astronomy, University of Hawaii, 2680 Woodlawn Drive. Honolulu, HI 96822 \\ SCOTT L. CuLLY \\ Space Sciences Laboratory, University of California, Berkeley, CA 94720 \\ Susana E. Deustua \\ Institute of Geophysics and Planetary Physics, Lawrence Livermore National Laboratory, Livermore, CA 94551 \\ Marek JablonsKi \\ Institute of Theoretical Astrophysics, University of Oslo, N-0315 Oslo, Norway \\ ChRISTOPHER M. JOHNS-KRULL \\ McDonald Observatory, University of Texas, Austin, TX 78712 \\ Bjørn R. Pettersen \\ Geodetic Institute, Norwegian Mapping Authority, N-3500 Honefoss, Norway \\ VERNE SMITH \\ Department of Astronomy, University of Texas. Austin, TX 78712 \\ William J. SPIESMaN \\ McDonald Observatory, University of Texas, Austin. TX 78712 \\ AND \\ JEFFREY VALENTI \\ Joint Institute for Laboratory Astrophysics, University of Colorado, Boulder. CO 80309 \\ Received 1995 March 10: accepted 1995 May 12
}

\begin{abstract}
We report on the first simultaneous Extreme-Ultraviolet Explorer $(E U V E)$ and optical observations of flares on the dMe flare star AD Leonis. The data show the following features:

1. Two flares (one large and one of moderate size) of several hours duration were observed in the EUV wavelength range:

2. Flare emission observed in the optical precedes the emission seen with $E U V E$;

3. Several diminutions (DIMs) in the optical continuum were observed during the period of optical flare activity.

To interpret these data, we develop a technique for deriving the coronal loop length from the observed rise and decay behavior of the EUV flare. The technique is generally applicable to existing and future coronal observations of stellar flares. We also determine the pressure, column depth, emission measure, loop crosssectional area, and peak thermal energy during the two EUV flares, and the temperature, area coverage, and energy of the optical continuum emission.

When the optical and coronal data are combined, we find convincing evidence of a stellar "Neupert effect" which is a strong signature of chromospheric evaporation models. We then argue that the known spatial correlation of white-light emission with hard X-ray emission in solar flares, and the identification of the hard $\mathrm{X}$-ray emission with nonthermal bremsstrahlung produced by accelerated electrons, provides evidence that flare heating on dMe stars is produced by the same electron precipitation mechanism that is inferred to occur on the Sun.

We provide a thorough picture of the physical processes that are operative during the largest EUV flare, compare and contrast this picture with the canonical solar flare model, and conclude that the coronal loop length may be the most important factor in determining the flare rise time and energetics.
\end{abstract}

Subject headings: stars: chromospheres - stars: flare - stars: individual (AD Leonis) - ultraviolet: stars

\footnotetext{
'NSF Young Investigator.
} 
, , 


\section{INTRODUCTION}

AD Leonis is a dMe flare star located in the immediate solar neighborhood, at a distance of about $5 \mathrm{pc}$. According to Pettersen, Coleman, \& Evans (1984), detectable flares (Johnson $U$-band enhancements of $\gtrsim 0.1 \mathrm{mag}$ ) occur every 100 minutes or so; more energetic flares occur with an exponential decrease in frequency. Since AD $L 00$ is the brightest active flare star visible from the northern hemisphere, it has been the target of several previous multiwavelength observing campaigns (e.g., Rodono et al. 1989; Hawley \& Pettersen 1991 [hereafter HP91]; Bookbinder, Walter, \& Brown 1992). We report here on a successful observing campaign using the NASA ExtremeUltraviolet Explorer ( $E U V E$ ) satellite and six ground-based optical telescopes. To our knowiedge these are the first simultaneous optical and EUV observations of stellar flares with the EUVE satellite.

The effort involved in organizing such an extensive observational campaign is more than justified by the importance of observing flare emission from both the high-temperature (coronal, X-ray) and low-temperature (chromospheric, optical) portions of the flaring plasma simultaneously. Previous investigations of this type are few and have reported varying results. Some workers have found that the coronal and chromospheric plasmas respond on a similar timescale to flare heating (e.g., Kahler et al. 1982; Haisch et al. 1983; de Jager et al. 1989), leading to the relations of Buller, Rodono, \& Foing (1988), Haisch (1989), and HP91 between the energy emitted in hightemperature (X-rays) and low-temperature (hydrogen and calcium lines, $U$-band continuum) flare features. However, other workers (Doyle et al. 1988; Haisch et al. 1981) found that there was no simultaneous response between the high- and low-temperature plasma during the flares that they observed.

Our goal was to obtain a new set of simultaneous coronal and chromospheric flare observations with higher spectral and temporal resolution than was previously attainable, and to analyze the data within the context of the generally accepted model for solar flares (e.g., Lin \& Hudson 1976; Dennis \& Schwartz 1989). In this moded, a flare begins with the rapid acceleration of a burst of nonthermal electrons down the magnetic field lines of a closed coronal loop (or an arcade of loops). The electrons collide with ions in the ambient plasma and emit hard X-rays via nonthermal bremsstrahlung, from which a power-law distribution can be inferred for the electron energy spectrum. White-light continuum emission is often observed from small kernels at the footpoints of flare loops. This emission is temporally and spatially correlated with the hard X-ray emission (see, e.g., Fig. 3.25 of Canfield et al. 1986; Hudson et al. 1992) and is therefore emitted at or near the sites where the accelerated electrons impact the preflare chromosphere and/or photosphere (although the exact emission mechanism remains unknown). The spiraling motion of the energetic electrons around the magnetic field lines also results in gyrosynchrotron emission at microwave frequencies. The nonthermal electrons heat the lower atmosphere, resulting in both emission from the low-temperature plasma and the "evaporation" of chromospheric plasma to coronal temperatures, filling the coronal loop with hot plasma. The term "evaporation" has been traditionally used to describe the "phase change" that occurs when dense, cool chromospheric plasma is heated during the flare and is transformed into hot, tenuous coronal plasma. As the corona increases in density (column depth), it becomes increasingly effective at stopping the energetic electrons, which now heat the corona directly, while the amount of heat deposited in the lower atmosphere diminishes. During this time the corona emits thermal soft X-rays. When the coronal heating ceases, the amount of material in the loop reaches its peak value and then gradually condenses back to its preflare configuration, while the soft X-ray flare emission dies away.

This canonical solar flare model manifests itself empirically through the well-known "Neupert effect" (Neupert 1968; Dennis \& Zarro 1993). Because chromospheric evaporation. which is responsible for increasing the coronal emission measure, is proportional to the flare heating rate, which is in turn proportional to the hard X-ray flux, one anticipates that a time integral of the hard X-ray flux should be proportional to the observed thermal soft X-ray flux. Dennis \& Zarro (1993) examined a data set of flares seen in both hard $X$-rays with the HXRBS spectrometer on SMM and in soft X-rays with the GOES satellite. They found that more than $80 \%$ of large events exhibit the Neupert effect. In this paper, we will use our optical data (as a proxy for hard X-rays) ${ }^{2}$ and $E U V E$ (essentially soft $X$-ray) data to search for a stellar flare analog to the Neupert effect.

In previous work we have quantified the overall theoretical framework of the canonical solar flare model by explicitly solving the energy equation for loops in the evaporating and condensing regimes (Fisher \& Hawley 1990, hereafter FH90). Given a prescribed flare heating rate, the loop evolution can then be determined. In this paper, we extend the analysis in FH90 so that the new EUVE data can be used to cietermine parameters such as the loop length, maximum column depth (a measure of the amount of evaporated material), and coronal pressure, as well as the flare area coverage, emission measure, and total thermal energy. We also use the techniques developed in Hawley \& Fisher (1992, hereafter HF92) to analyze the optical flare emission and to determine the temperature, area coverage, and total energy deposited in the lower atmosphere. The optical and coronal results are then combined to investigate properties of the flare heating mechanism and the efficacy of the solar flare picture in describing the observed stellar flares.

\section{THE DATA}

As part of the first year of the $E U V E$ guest investigator program, we obtained EUV observations of AD Leo over 42 satellite orbits between 1993 March 1-3. The data consist of spectra in the short-, medium-, and long-wavelength regions, plus EUV " photometry " obtained with the Deep Survey (DS) instrument. We defer discussion of the spectra to a later paper (Cully et al. 1995) and concentrate here on the DS data. To complement the EUV observations and provide information on the chromospheric flare response, we organized a simultaneous optical campaign, which resulted in data from six optical telescopes: the Lick Observatory 24 inch $(0.61 \mathrm{~m})$ (photometry), 40 inch (1.02 m) (spectroscopy), and 24 inch $(0.61$ m) Coude Auxiliary Telescope (echelle spectroscopy); the McDonald Observatory 36 inch $(0.92 \mathrm{~m})$ (photometry) and 82 inch $(2.08 \mathrm{~m})$ (echelle spectroscopy); and the 100 inch $(2.54 \mathrm{~m})$ Nordic Optical Telescope (spectroscopy).

\footnotetext{
2 With existing telescopes, hard X-ray emission is unobservable from stellar flares, even for the nearest stars, because nonthermal bremsstrahlung is such an inefficient process. While impulsive gyrosynchrotron emission from highly energetic electrons may have been detected in a few stellar ftares (see $\$ 4.3$ o the review by Bastian 1990: Fig. I of Rodono et al. 1989), the white-ligh continuum is the only easily measurable tracer of the impulsive energy releas on flare stars, if we adopt the solar model.
} 


\subsection{Observational Ensemble}

Table 1 is an observing $\log$ for the seven telescope/ instrument combinations including the observers, type of data obtained, and observing intervals. We discuss each individually below.

\subsubsection{EUVE/DS}

Malina \& Bowyer (1991) provide a description of the EUVE. Briefly, the telescope has a $2: 1$ diameter field of view and an on-axis angular resolution of $\sim 18^{*}$. A specific target is visible for some $\mathbf{3 0}$ minutes of each 90 minute orbit, with visibility defined by Earth occultation and the geocoronal background count rate. The Deep Survey (DS) instrument is also described in Malina \& Bowyer (1991) and in Siegmund et al. (1986). We used the Lexan/Boron filter, which has a bandpass of 65-190 A at $10 \%$ of peak transmission. The spectroscopic data (Cully et al. 1995) show that the strongest lines in this bandpass are mainly from highly ionized stages of $\mathrm{Fe}$ formed at temperatures of $10^{6}$ to several $\times 10^{7} \mathrm{~K}$.

The DS data were processed into files containing all counts detected within $12^{\prime}$ of the target position, along with the arrival time, detector location, and aspect-corrected sky position of each photon. The average source count rate was higher than the local sky and detector background rates, so the source location could be determined visually. We determined that the spacecraft pointing during our AD Leo observation was stable to within $5^{\prime \prime}$ over more than $90 \%$ of our exposure time, and the occasional short deviations (all $<70^{\circ}$ ) that did occur took place when AD Leo was in a state of low activity. A symmetrized version of the DS point spread function was used to calculate the source and background count rates. The data were then corrected for instrumental deadtime and "Primbsch" effects (Abbott, Miller, \& Gavin 1993) and binned into $500 \mathrm{~s}$ intervals. The optically bright binary giant, $\gamma$ Leo (K0 III + G7 III), lies 6' to the SE of AD Leo. We have examined a deep, 26 ks exposure of AD Leo taken with the PSPC instrument aboard ROSAT (image rp200076) which we obtained from the ROSAT archives, and we find that neither of the late-type giant stars is present as a soft $\mathrm{X}$-ray source. Therefore we attribute all the EUVE emission we have observed to AD Leo.

A possible source of uncertainty in the DS count rate is introduced by an area of decreased sensitivity in the center of the DS microchannel plate detector that appeared after an extensive observation of the white dwarf HZ 43 in 1993 February (Miller 1994). This "dead spot" is centered on the bore site of the instrument, with a maximum loss of efficiency in some channels of $\sim 80 \%$. To determine whether the dead spot affected the DS count rate in our observation, we compared the wavelength-integrated count rate in the SW detector of the EUVE spectrograph with the DS count rate and found that the count rates are consistent with one another if no dead spot correction is applied to the DS data. We also compared the ratio of the DS count rate to the SW count rate from the observation of AU Mic (made before the observation of $\mathrm{HZ} 43$ ) (Cully et al. 1993, 1994) to the same ratio from our AD Leo observations and found that it did not change, further indicating that the DS count rate was not strongly affected by the dead spot during our AD Leo observations. Therefore we have not applied any dead spot correction to our DS count rates for AD Leo. If it becomes clear at a later date that the count rates we report here need to be changed by a "correction factor" $\int_{d v}$, then some of the coronal parameters that we derive in $\S 4.2$ for the flare observations will be affected. The footnotes to Tables 2 and 3 describe how the quoted values scale with $f_{\text {da }}$. The value $f_{a}=1$ adopted here is probably known only to within a factor of 2 or so based on the tests we have performed. Values of $f_{d}$ as large as 3-4 (i.e., corresponding to the possible maximum loss of efficiency of $\sim 80 \%$ ), while changing some of the coronal parameters, will not significantly affect our conclusions.

\subsubsection{Photometry}

Photometric observations were made at the Lick Observatory 24 inch telescope and the McDonald Observatory 36 inch telescope. The photometry at Lick was obtained with a single-channel photometer, which was equipped with standard Johnson $U, B$, and $V$ filters and a $1 \mathrm{P} 21$ photomultiplier cooled with dry ice. Because the filters had to be changed manually, AD Leo was monitored only in $U$, with occasional observations in $B$ and $V$. The integration time was $2 s$ in each filter. The observing periods were photometric, and the data were corrected for extinction and reduced to the standard system using observations of standard stars (Landolt 1973, 1992).

At McDonald, a two-star photometer was used for UBV photometry. The primary photomultiplier was thermoelectrically cooled, while the secondary tube was operated at ambient temperature. AD Leo was observed through the primary

TABLE 1

ObserviNG LOC

\begin{tabular}{|c|c|c|c|c|}
\hline $\begin{array}{l}\text { Telescope/ } \\
\text { Observers }\end{array}$ & Instrument & $\begin{array}{l}\text { Wavelengths/ } \\
\text { Resolution }\end{array}$ & Time (UT) & $\begin{array}{l}\text { Elapsed } \\
\text { Time (hr) }\end{array}$ \\
\hline EUVE/Simon ................. & Deep Survey & $65-190 A$ & $\begin{array}{l}\text { Mar } 16.0- \\
\text { Mar } 323.3\end{array}$ & $6.0-71.3$ \\
\hline Lick 24 inch/Johns, Hawley......... & photometer & $U(B V)$ & $\begin{array}{l}\text { Mar } 17.8-9.5 \\
\text { Mar } 25.0-12.1\end{array}$ & $\begin{array}{c}7.8-9.5 \\
29.0-36.1\end{array}$ \\
\hline Lick 40 inch/Deustua, Hawiey....... & $\begin{array}{l}\text { medium-resolution } \\
\text { spectrograph }\end{array}$ & $\begin{array}{l}3900-7000 A \\
S A\end{array}$ & Mar 2 7.4-11.7 & $31.4-35.7$ \\
\hline $\begin{array}{l}\text { Lick CAT/Cully, Valenti ............. } \\
\text { McDonald } 36 \text { inch/Spiesman ........ }\end{array}$ & $\begin{array}{l}\text { Hamilton echelle } \\
\text { spectrograph } \\
\text { photometer }\end{array}$ & $\begin{array}{l}4800-8700 A \\
R=48,000 \\
U B V\end{array}$ & $\begin{array}{l}\text { Mar } 15.0-12.0 \\
\text { Mar } 23.5-12.5 \\
\text { Mar } 28.3-10.3 \\
\text { Mar } 35.9-10.5\end{array}$ & $\begin{array}{r}5.0-12.0 \\
27.5-36.5 \\
32.3-34.3 \\
53.9-58.5\end{array}$ \\
\hline $\begin{array}{l}\text { McDonald } 82 \text { inch/Smith } \\
\text { NOT } 100 \text { inch/ }\end{array}$ & $\begin{array}{l}\text { Sandiford echelle } \\
\text { spectrograph }\end{array}$ & $\begin{array}{l}6000-8000 \AA \\
R=60,000\end{array}$ & $\begin{array}{l}\text { Mar } 29.7,11.3 \\
\text { Mar } 36.2-7.0\end{array}$ & $\begin{array}{l}33.7,35.3 \\
54.2-55.0\end{array}$ \\
\hline Pettersen, Jabionski ................ & $\begin{array}{l}\text { medium-resolution } \\
\text { spectrograph }\end{array}$ & $\begin{array}{l}3900-4900 \AA \\
3.8 \AA\end{array}$ & $\begin{array}{l}\text { Mar } 20.7-4.6 \\
\text { Mar } 2 / 3 \quad 21.5-1.4 \\
\text { Mar } 35.9-6.6\end{array}$ & $\begin{array}{l}24.7-28.6 \\
45.5-49.4 \\
53.9-54.6\end{array}$ \\
\hline
\end{tabular}


channel, and the second channel was used to monitor sky conditions. An integration time of $2 \mathrm{~s}$ was used at each of the $U, B$, and $V$ filter positions. An automatic filter wheel and computercontrolled integrations provided a total cycle time of $7 \mathrm{~s}$. The sky was photometric for most of the observing period, although high winds forced occasional temporary closures of the dome. As with the Lick data, Landolt standards were used to correct for atmospheric extinction and to reduce the data to the standard $U B V$ system. The absolute photometric measurements at the two sites agreed to within $\sim 0.02 \mathrm{mag}$ in each filter during periods of simultaneous observing, indicating external agreement at the level of $\sim 2 \%$.

\subsubsection{Medium-Resolution Spectroscopy}

Medium-resolution spectroscopy was obtained with the Lick 40 inch Nickel Telescope and with the 100 inch Nordic Optical Telescope (NOT) at La Palma. The Lick observations were made with a Cassegrain Spectrograph equipped with a 600 lines $\mathrm{mm}^{-1}$ grating, giving an effective resolution of $2.6 \AA$ pixel $^{-1}$ on a $400 \times 1200$ Reticon CCD detector. The slit width was $3^{\prime \prime}$, corresponding to 2 pixels on the CCD. The spectra cover the wavelength region $3900-7000 \AA$. Exposure times varied from 20 to $30 \mathrm{~s}$, with a minute between successive exposures for readout and recording. Calibration frames included an argon-neon arc lamp for wavelength calibration and spectra of standard stars from Massey et al. (1988) for flux calibration. The data were reduced and transformed to a calibrated flux system using IRAF. We expect that there were small ( $\$ 5 \%)$ light losses from the edges of the slit as a result of seeing changes and guiding errors. The night was photometric.

The observations on the NOT were made with the Low Dispersion Spectrograph. The spectra cover the wavelength region from 3900 to $4900 \AA$ with an effective resolution of $1.9 \AA$ pixel $^{-1}$. The slit width was 1.1 which corresponded to 2 pixels on a thinned, back-illuminated Tektronix CCD. The exposure time was $20 \mathrm{~s}$, with $5 \mathrm{~s}$ between successive exposures for readout and recording. The data were reduced using routines written in the Interactive Data Language (IDL). The weather was not photometric, so only the equivalent widths of the principal emission lines were obtained.

\subsubsection{Echelle Spectroscopy}

High-resolution spectra were obtained in the 4800-8700 $\AA$ region at Lick Observatory with the 24 inch Coude Auxiliary Telescope (CAT) and in the $6000-8000$ A region at McDonald Observatory with the 82 inch Struve Telescope. The CAT observations employed the Hamilton Echelle Spectrometer and a cooled TI $800 \times 800 \mathrm{CCD}$ (Vogt 1987). The spectrometer was designed for use with a $2048 \times 2048$ CCD; thus, use of the TI $800 \times 800 \mathrm{CD}$ resulted in gaps in the spectral coverage. The resolving power of the spectrometer is approximately $R=\lambda / \Delta \lambda=48,000$; there were 2.5 pixels per resolution element. The slit width of $500 \mu \mathrm{m}$ that was used corresponds to 1.5 pixels on the detector and 4.7 on the sky. The data were reduced using the IDL procedures outlined by Basri, Wilcots, \& Stout (1989) and Valenti (1994). Exposure times were 15 minutes per frame during quiescence and 5 minutes per frame during flare. The readout time was approximately 1 minute for each exposure.

Observations at McDonald were made with the Sandiford cross-dispersed echelle spectrometer (McCarthy et al. 1993) at the Cassegrain focus. The detector is a Reticon $400 \times 1200$ CCD with $27 \mu \mathrm{m}$ pixels; the two-pixel resolution is 60,000 . The two-dimensional raw frames were reduced to single-order one- dimensional spectra using the IRAF echelle data reduction routines. Integration times were 2 minutes, with each extracted spectrum having a signal-to-noise ratio of about 40 in the “continuum" at $\mathrm{H} \alpha$.

\subsection{EUV and Optical Flare Observations}

Figure 1 shows the EUV light curve obtained with the $E U V E / D S$ instrument. Each point represents an independent bin of $500 \mathrm{~s}$ of integration time. The time axis is measured in hours from 1993 March 1.0 UT. Throughout the paper, we will use this time axis as a reference for all the data, including the optical (see col. [5] of Table 1). Also indicated in the lower half of Figure 1 are the observing intervals for the optical data. It is clear that two major EUV flares occurred during the time we were monitoring the star. The first (hereinafter EUV flare 1, or EF1) began between $33: 30$ and $34: 30 \mathrm{hr}$, reached a peak count rate of $\sim 1.0$ counts $\mathrm{s}^{-1}$, and continued for at least $8 \mathrm{hr}$, while the second (hereinafter EUV flare 2, or EF2) began between 55:30 and $56: 30 \mathrm{hr}$, reached a peak count rate of $\sim 0.8$ counts $\mathrm{s}^{-1}$, and continued for approximately $3 \mathrm{hr}$. Note that EF2 occurred while the DS count rate was still elevated from the decay of EF1. We were fortunate to have optical coverage which overlapped both flares, during the time intervals denoted in Figure 1 by $C(E F 1)$ and $E(E F 2)$. There are three other intervals with simultaneous optical and EUV observations, and these are labeled $A, B$, and $D$ in the same figure.

Figures $2 a-2 e$ show the data obtained during the five simultaneous observing periods, A-E. Period $A$ is a time of relative quiescence, while $B$ and $D$ show small $H \beta$ flares with little or no EUV response. Note that we are unable to resolve low-level flare enhancements in the EUV data because of the possible small-scale variability introduced by the presence of the dead spot. Thus, we can make no quantitative statements about the presence or absence of low-level, short-lived EUV emission such as might be expected to accompany these small optical flares.

The first large EUVE flare, EF1, begins during period $\mathrm{C}$. Both photometric sites observed a $U$-band flare beginning at 32:58 hr, which reached a peak brightness some $0.7 \mathrm{mag}$ above the quiescent level, followed by a sharp and then more gradual decline. The $U$-band flux did not return to its quiescent level, but remained enhanced for several hours. A second $U$-band flare occurred at $35: 48 \mathrm{hr}$, peaking $0.6 \mathrm{mag}$ above the preflare level, or equivalently $0.8 \mathrm{mag}$ above the quiescent level. Our medium-resolution spectroscopy shows that the hydrogen lines followed the $U$-band flare in the expected way, increasing in fux during the onset of the initial flare, followed by a slow decline. Figure $2 c$ (bottom panel) gives the $\mathrm{H} \gamma$ light curve at a time resolution of 5 minutes. We also have data for the other Balmer lines, which follow the same time evolution as $\mathrm{H} \gamma$, and for $\mathrm{Ca}$ II $\mathrm{K}$, which is quite noisy but appears to have a somewhat slower evolution, in accord with the results in HP91. The Balmer decrement at the peak of the first $U$-band flare (preceding EF 1) is consistent with the decrement obtained by HP91 and hence, as shown there, with other stellar flares. The flare decrement is quite shallow compared to the quiescent decrement, indicating that the luminosity in the higher Balmer lines increases markedly compared to $\mathrm{H} \alpha$.

The Balmer decrement provides an observational constraint on chromospheric models (such as those in Houdebine \& Panagi 1990; HF92; Houdebine \& Doyle 1994a, b), particularly when information on the flare heating, through optical continuum and high-energy observations, is available. In solar 


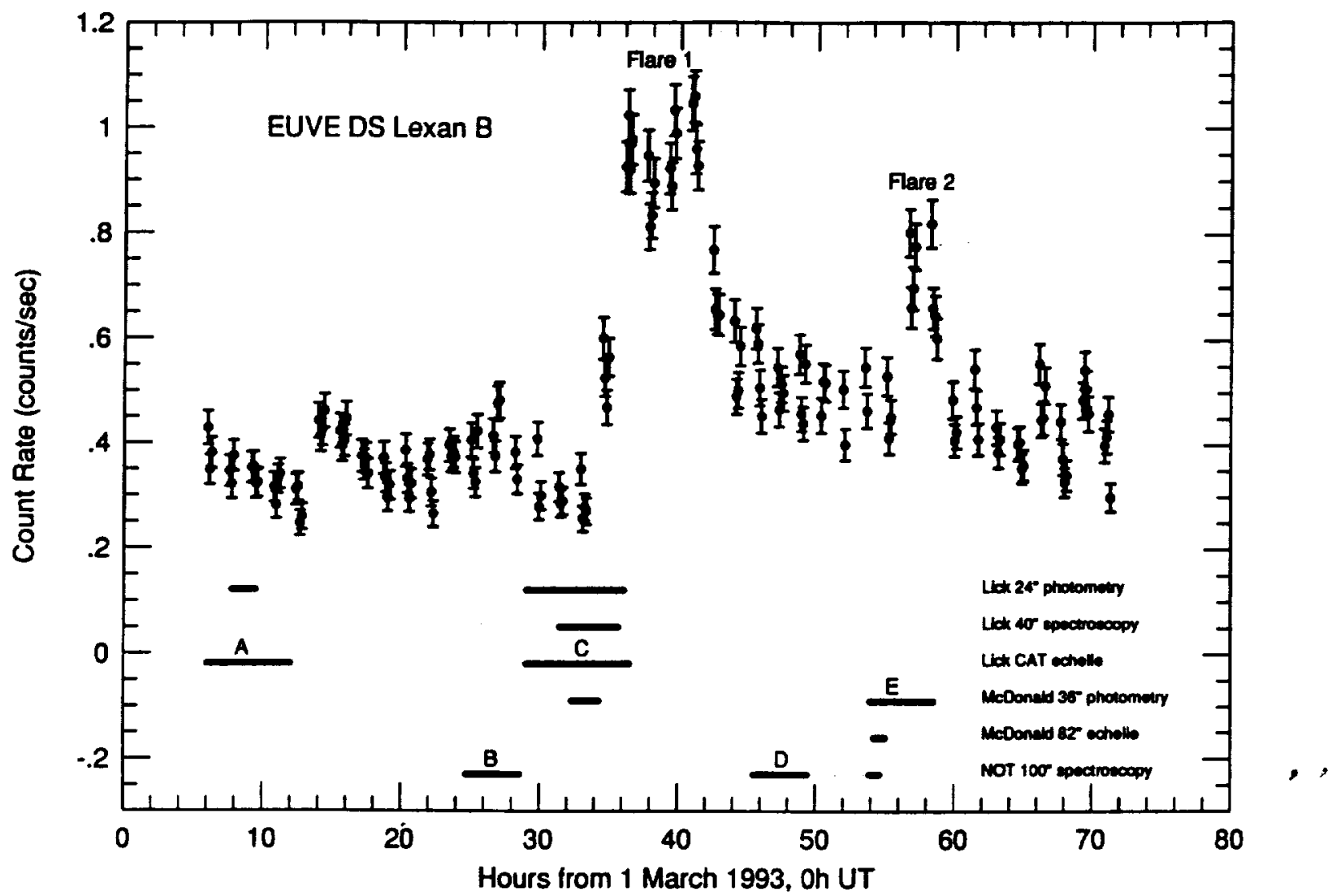

FIG. 1.-EUVE/DS light curve for the entire period of observation, with time (in hr) measured from 1993 March 1.0 UT. Horizontal bars at the bottom of the plot indicate the times when coordinated observations were being made from each of the ground-based telescopes in the campaign. Periods of overlapping observations are labeled $\mathrm{A}-\mathrm{E}$, in chronological order.

flares, $\mathrm{H} \alpha$ becomes greatly enhanced. Stellar flares may thus differ from their solar counterparts in this regard. To determine whether such behavior reflects merely a difference in atmospheric structure, or a more fundamental change in the type and/or amount of chromospheric flare heating, will require more detailed modeling of the flare chromosphere.

Unfortunately, the star set at Lick shortly after the second optical flare, and we have no further optical data for the duration of EF1.

The top panel in Figure $2 c$ shows the EUVE/DS data during the overlapping time period. It is immediately apparent that EF1 is not coincident with the beginning of the optical activity. In fact, the EUVE orbit spanning 32:50-33:20 hr exactly covers the onset of the first optical flare, and no simultaneous EUV flare was observed. The minimum time delay between the optical and EUV flares is $\sim 25$ minutes, corresponding to the end of this orbit, when EUV data were no longer being acquired. Clearly by the start of the next orbit (at $34: 30 \mathrm{hr}$ ), the EUV count rate was enhanced, representing the first data obtained during the rise phase of EF1. The first noteworthy result of this work is, therefore, the considerable delay between the rise in optical light (both continuum and emission lines) and in the EUV photometry. A peak in the DS data is seen during the orbit near $36: 15$, and for the next several hours the flare emission hovers near this peak value. A sharp decay from the flare peak is clearly visible in the orbit near $42: 00$, and a change to a shallower decline occurs near 44:00, where the DS counts are still elevated above the quiescent value.

Flare EF2 occurred during period E (Fig. $2 e$ ). Just after 54:00 hr, an optical flare is seen in both the $U$-band and the
$H \beta$ equivalent width. The $H \alpha$ data begin shortly after the flare peak but are enhanced. Another $U$-band flaring episode begins at $55: 25 \mathrm{hr}$ and continues with short timescale variability for at least $3 \mathrm{hr}$ until the end of the observations. Overlapping this optical activity, EF2 begins sometime between $55: 30$ and $56: 30 \mathrm{hr}$, reaches a peak that lasts at least $1.5 \mathrm{hr}$ between $56: 30$ and 58:00, and decays by 60:00 hr. There is not a clearly measurable time lag between the optical and EUV emission in $\mathrm{EF} 2$, possibly because of our inability to resolve the EUV data on the shorter timescales that are involved.

In Table 2 we compile the empirical results from the observations, including the rise and decay phase timescales, count rates, average magnitudes, and total (time integrated) energies for both the optical and EUV passbands in each flare. The EUV energy was computed from the count rate by assuming an average photon wavelength of $100 \AA$ and an effective EUVE/DS detector area of $25 \mathrm{~cm}^{2}$ (Cully et al. 1994, hereafter CFAS, Fig. 2), while the $U$-band energy comes from the measured magnitudes and the usual Johnson (1966) calibration. Note that since our $U$-band data fail to cover the entire period of the EF1 flare, our energy estimate is a lower limit to the total $U$-band energy radiated over the course of the flare event. Both the optical and EUV energy estimates assume that the flare radiation is emitted isotropically from a source that is unocculted.

\section{DISCUSSION OF THE OPTICAL DATA}

The optical continuum of AD Leo, as measured by the $U$-band photometry, shows long-lived flaring episodes during both EUV flares. In most previous work on stellar flares, the 

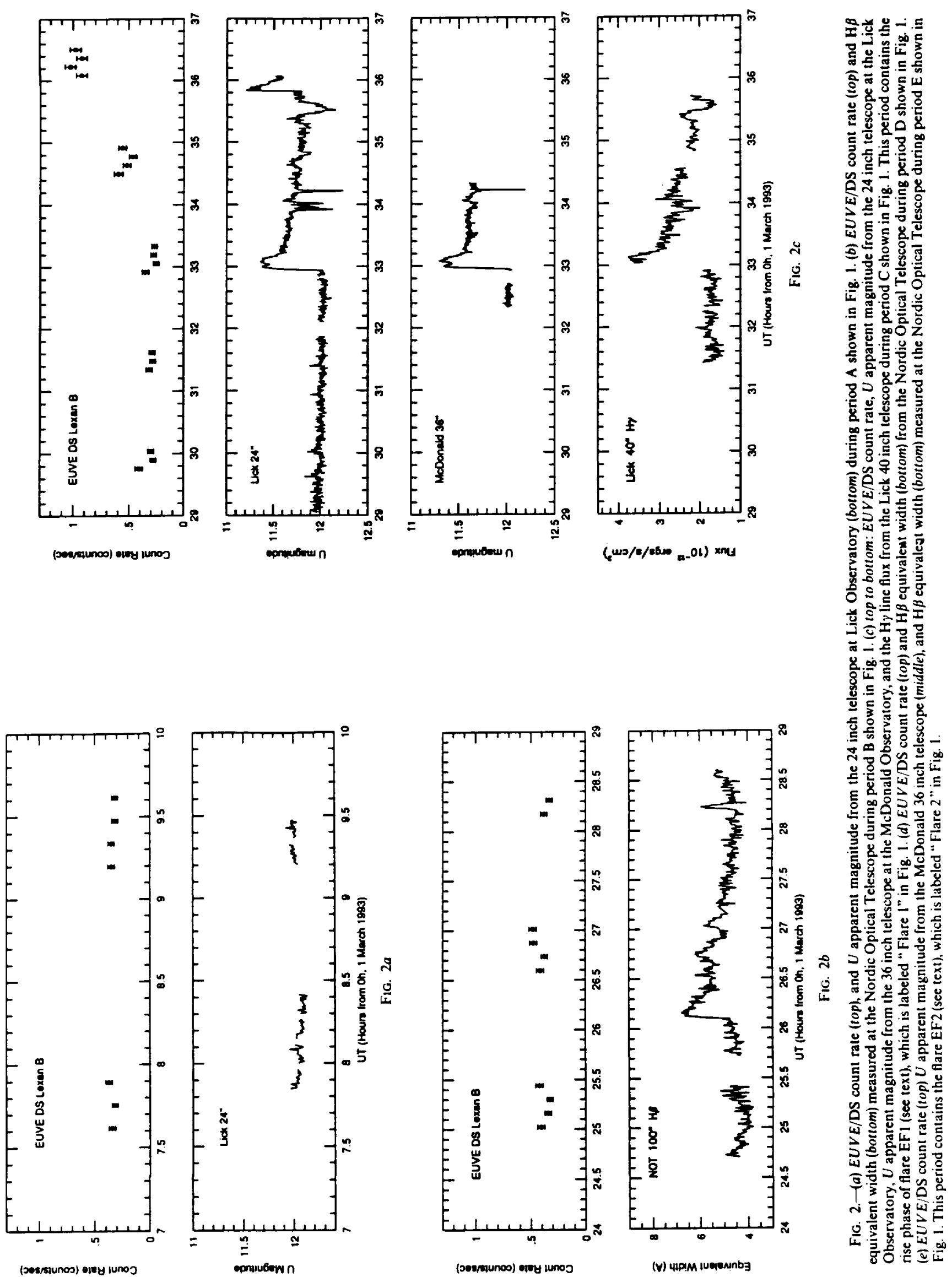

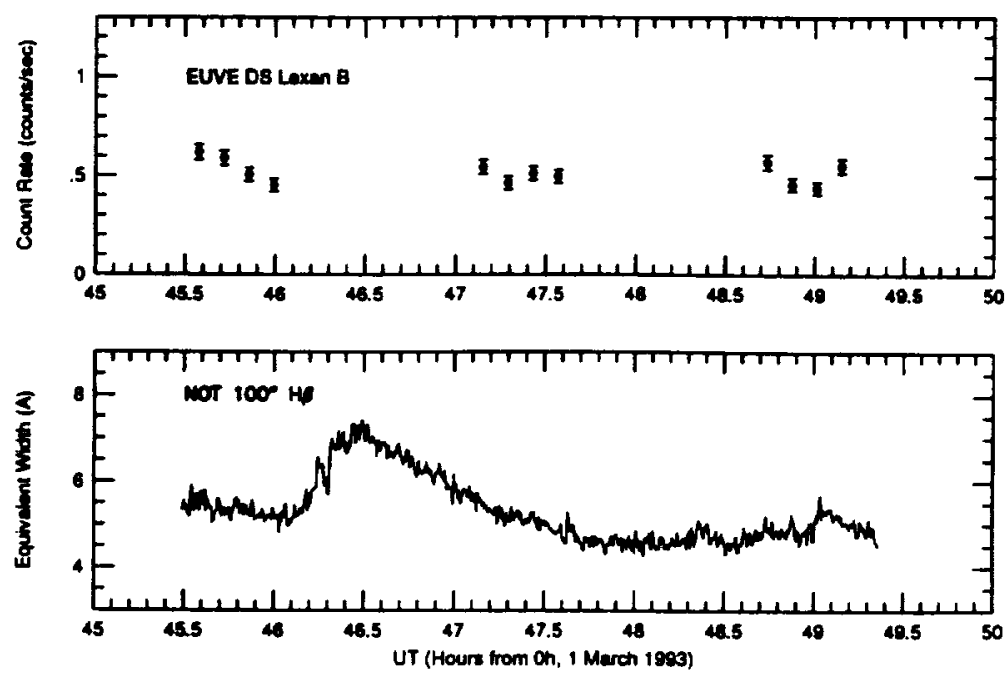

Fig. $2 d$
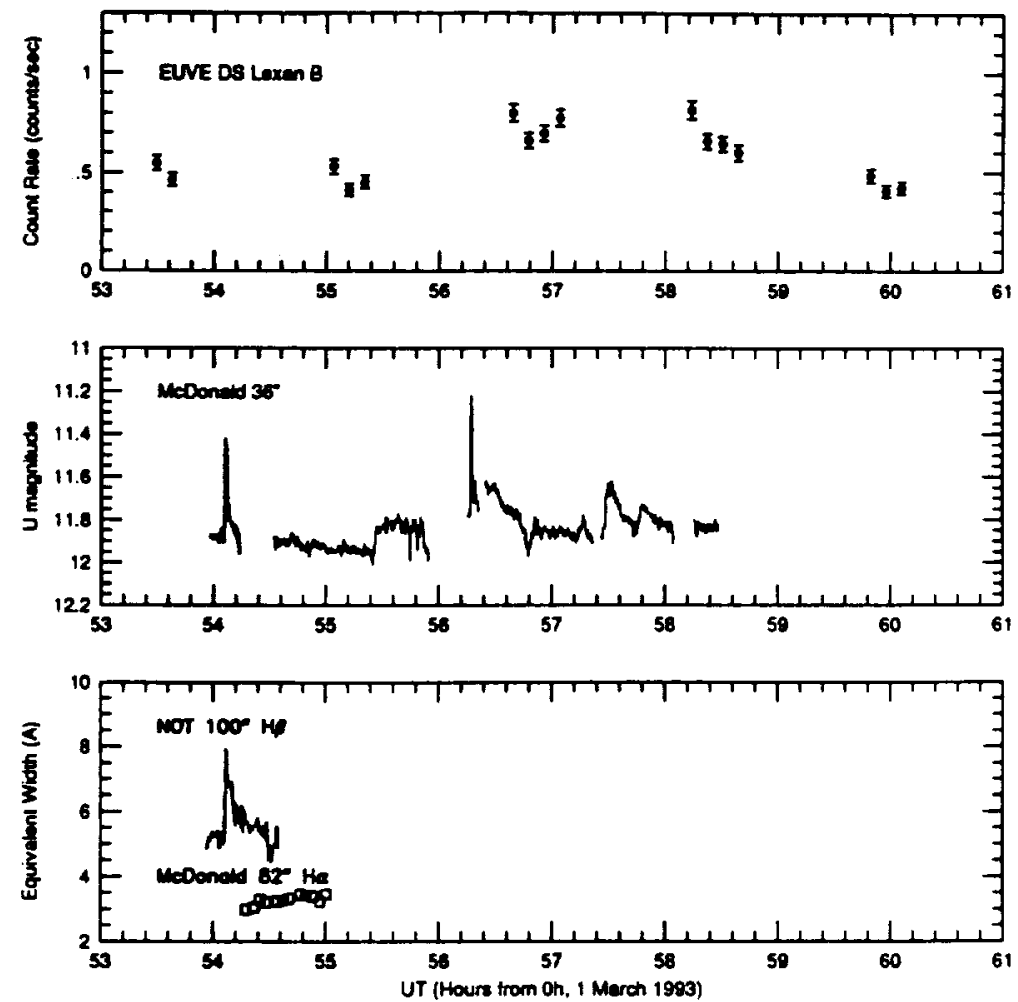

Fig. $2 e$

"canonical" $U$-band light curve has a rapid rise of some seconds to a few minutes, which is followed by a slower, exponential decline lasting some tens of minutes (Moffett \& Bopp 1976). In general there is also a correlation between the rise and decay times and the flare energy, in the sense that more energetic flares take longer to rise and decay. The flares reported here do not follow such a neat picture. The two distinct optical flares observed during period C (accompanying EF1) do show rapid rise times, but the decay from the first peak is interrupted, remaining elevated at $\sim 0.2$ mag above the quiescent level for almost $3 \mathrm{hr}$ before rising to the second peak. The majority of the flare energy is emitted not during the peak, but during the long period of decline. Such behavior, though unusual, is by no means unprecedented. Long-lived, low-level flaring episodes have been reported previously by a number of authors (e.g., Byrne 1979, Fig. 3; Moffett 1974, Tables 17-23; Pettersen et al. 1986, Table III and Fig. 5). Also, there has been a tendency in the past to ignore continuing low-level emission after the main flare peak has passed (see, e.g., Moffett \& Bopp 1976, Figs. 1-3), so that extended periods of activity may actually be more common than is generally thought. The advantage of having two photometric telescopes observing simultaneously during period $\mathrm{C}$ makes our observations unequivocal; the excess emission cannot be ascribed simply to 
TABLE 2

MEASURED QUANTTIES

\begin{tabular}{|c|c|c|c|}
\hline Quantity & EF1 & EF2 & AU Mic \\
\hline \multicolumn{4}{|c|}{ EUV } \\
\hline 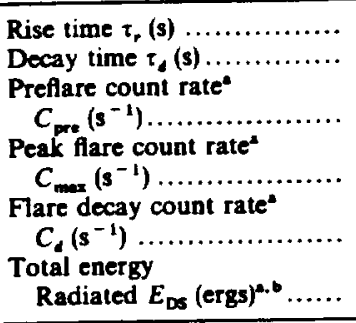 & $\begin{array}{c}11000 \\
6000 \\
0.27 \\
0.98\end{array}$ & $\begin{array}{c}<6000 \\
<6000 \\
0.46 \\
0.76 \\
0.46: \\
8.2 \times 10^{31} \\
\end{array}$ & $\begin{array}{r}5600 \\
4200 \\
0.50 \\
7.00 \\
4.30 \\
3.9 \times 10^{33} \\
\end{array}$ \\
\hline \multicolumn{4}{|c|}{ Optical } \\
\hline 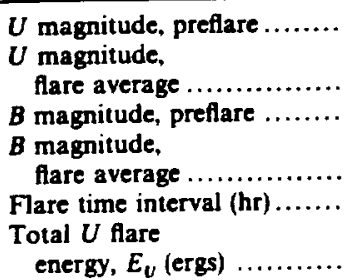 & $\begin{array}{c}12.00 \\
11.70 \\
10.98 \\
10.90 \\
32.9-36.1 \\
>4.9 \times 10^{32}\end{array}$ & $\begin{array}{c}10.93 \\
55.4-58.4 \\
26 \times 10^{32}\end{array}$ & $\begin{array}{l}\cdots \\
\cdots\end{array}$ \\
\hline
\end{tabular}

- The count rates and DS energy listed here assume no correction for possible "dead spot " effects in the AD Leo observation. If a correction factor $f_{\Delta}$ is applied to the DS count rate, each quantity should be multiplied by $f_{\text {dur }}$

${ }^{6} E_{\text {s }}$ is the total energy radiated during the rapid rise, flare peak. and rapid decay phases which we model in this paper. It does not include the energy radiated during the long slow decay phase seen after the rapid decay phase in $\mathrm{EF} 1$ and the AU Mic flare.

instrumental effects. In fact, careful optical monitoring might reveal that activity of this type is actually prevalent on dMe stars. The two optical flare events accompanying EF1 and EF2 each have total $U$-band energies of $\gtrsim 10^{32}$ ergs (see Table 2); thus, they are more energetic than $\sim 95 \%$ of the flares studied by Pettersen et al. (1984).

The detailed $U$-band flare light-curve morphology undoubtedly contains interesting information about the flare heating in the deep atmosphere. Unfortunately, until we can identify and model the exact mechanism by which the $U$-band continuum is produced, we are unable to use the detailed lightcurve morphology to study flare heating in a quantitative way. We do, however, use the overall shape and energy to investigate the Neupert effect during stellar flares (see $§ 5$ ).

\subsection{Diminutions in the Stellar Continuum (DIMs)}

Figure $2 c$ shows several other intriguing features in the continuum behavior that are corroborated by observations at more than one telescope. Diminutions in the stellar continuum appear at times $33: 52,34: 15$, and $35: 27$. These diminutions (or DIMs, as we choose to call them) may be similar to the "dips" first described by Cristaldi, Gershberg, \& Rodono (1980), and also reported by Giampapa et al. (1982), Doyle et al. (1988), and Rodono et al. (1989). Haisch et al. (1983) discussed several empirical explanations for these features. Grinin (1983) suggested that such features may be produced when the initial burst of flare energy impacts the photosphere, leading to a sudden rise in the $\mathrm{H}^{-}$opacity and momentarily upsetting the radiative equilibrium. Henoux et al. (1990) discussed possible "black light flares" on the Sun using a similar model. Gurzadyan (1988) proposed a way to distinguish DIMs observationally by observing both the blue and red response-the
near-IR flux should become brighter during the time that the blue flux is diminished. Interestingly, the reverse of this phenomenon (IR dips during $U$-band enhancements) is seen in some flare observations (Rodono et al. 1989). Geometric effects (e.g., prominences blocking part of the stellar surface) or even instrumental problems cannot be entirely ruled out. We are confident that the long-lasting diminutions at $\sim 33: 52$ and $\sim 35: 27$ are real, but the short, deep excursion at $\sim 34: 15$ seen at both photometric sites might be coincidental, since the shape and timing of the event differ when the two sets of data are examined in detail. The fact that both longer lived diminutions occurred just prior to optical flares may also be important and fits with both previous observations and Grinin's theory.

\subsection{Temperature, Area Coverage, and Energy}

In HF92 we analyzed the continuum emission from the very large 1985 April 12 flare observed on AD Leo and showed that the optical continuum observed in four bandpasses $(U, B, V$, and $R$, as well as the UV continuum seen in the SWP and LWP bandpasses on the International Ultraviolet Explorer (IUE), could be fitted by a blackbody spectrum at a temperature of $\sim 9000 \mathrm{~K}$, with only a small area coverage factor of the star. Other proposed continuum emission mechanisms. including free-free emission and hydrogen recombination radiation, could not explain the observed continuum spectrum. Several other authors (Mochnacki \& Zirin 1980;'Giampapa 1983; Kahler et al. 1982) have also fitted a blackbody spectrum to optical flare continuum emission. The current data for AD Leo include $U$ and $B$ observations during the optical flares (the $V$ band is not significantly enhanced relative to the quiescent flux for these flares), which are insufficient to make an independent determination of the emission mechanism. Thus, we assume that the emission comes from a blackbody source, as in the previous flares, and we solve for the temperature and area coverage of the source.

The quiescent energy fluxes observed at earth in the $U$ and $B$ bandpasses prior to a flare are designated by $F_{U_{0}}$ and $F_{B_{0}}$. We assume that during the flare a fraction $X$ of the stellar area emits as a blackbody at temperature $T_{f 1}$. The flux radiated in the $U$ and $B$ bandpasses by the flaring areas of the star is then $\pi B,\left(T_{i}\right)$, convolved with the respective filter response functions. If $F_{U}\left(T_{n}\right)$ and $F_{B}\left(T_{f}\right)$ are the fluxes in the $U$ and $B$ bandpasses that would be observed at Earth if the entire star were at temperature $T_{n}$ (the convolutions described above diluted by the factor $R_{*}^{2} / d^{2}$ ), then the increases in the $U$ and $B$ fluxes $\Delta F_{V}$ and $\Delta F$ observed at Earth during the flare can be written

$$
\Delta F_{U}=X F_{U}\left(T_{\mathrm{f} 1}\right)-X F_{U_{0}},
$$

and

$$
\Delta F_{B}=X F_{B}\left(T_{10}\right)-X F_{B_{0}} .
$$

These equations do not include possible projection effects or partial occultation of the flaring area behind the limb, and so the derived area coverage, $X$, should be used with appropriate caution.

A transcendental equation for the flare temperature $T_{f l}$ can be found by dividing the two equations, resulting in

$$
\frac{\Delta F_{B}}{\Delta F_{U}}=\frac{F_{B}\left(T_{11}\right)-F_{B_{0}}}{F_{U}\left(T_{\mathrm{f}}\right)-F_{U_{0}}}
$$

Once $T_{f 1}$ has been determined from equation (3.3), the coverage factor $X$ can be found from either equation (3.1) or (3.2). 
The quiescent magnitudes for AD Leo (see Table 2) are $U_{0}=12.00$ and $B_{0}=10.98$. During the rise phase of EF1, the average flare magnitudes are $U_{n} \sim 11.70$ and $B_{f 1} \sim 10.90$. Equation (3.3) then gives $T_{\mathrm{r}} \sim 9000 \mathrm{~K}$ (compatible with estimates made for other flares) and $X \sim 1.0 \times 10^{-4}$. An estimate of the total flare optical luminosity is then given by $L_{o p t}=\sigma T_{f 1}^{4}$ $\times X \times 4 \pi R_{*}^{2} \sim 4 \times 10^{29} \mathrm{ergs} \mathrm{s}^{-1}$. The flare emission continues for at least $3.2 \mathrm{hr}$ (when the observations ended), so a lower limit to the optical flare energy is $E_{\text {opt }} \geq 4.6 \times 10^{33} \mathrm{ergs}$, or about 10 times the measured $U$-band energy (Table 2). Flare EF2 has average flare magnitudes of $U_{n} \sim 11.82$ and $B_{11} \sim$ 10.93 , giving $T_{\mathrm{n}} \sim 9500 \mathrm{~K}$ and $X \sim 5.3 \times 10^{-5}$, and $L_{\text {opt }} \sim 2.5$ $\times 10^{29} \mathrm{ergs} \mathrm{s}^{-1}$. In this case, we estimate an optical flare duration of $3.1 \mathrm{hr}$, from which we find $E_{-2} \sim 28 \times 10^{33} \mathrm{ergs}$, again approximately 10 times the $U$-band energy in Table 2 . These derived optical flare quantities are given in Table 3 , along with the coronal properties that we obtain from the models in the next section.

\section{EUV DATA AND CORONAL LOOP MODELS}

Using the EUV flare data presented in $\$ 2$, we now seek to apply elements of our flare loop evolution model to determine the physical parameters of the coronal loops within which flares EF1 and EF2 occurred. First we review the basic precepts of the model and derive expressions relating observed quantities (such as the flare rise and decay times) to the model parameters (such as the loop length). Then we analyze flares $\mathrm{EF} 1$ and EF2 and discuss the resulting loop parameters with reference to previous work.

\subsection{Flare Loop Evolution}

In FH90, we solved an energy equation for a coronal loop in the evaporating and condensing regimes. In order to use an analytic expression for the energy balance in the loop, it was necessary to make several basic assumptions about the loop conditions and geometry. Our model is strictly valid only when these assumptions are realized. The assumptions are as follows: (1) The gas pressure is small compared to the magnetic pressure within the loop; (2) Flare timescales are long compared with the acoustic transit time along a coronal loop, so

TABLE 3

DeRIVED QUANTITL

\begin{tabular}{|c|c|c|c|}
\hline Quantity & EFI & EF2 & AU Mic \\
\hline \multicolumn{4}{|c|}{ Optical } \\
\hline 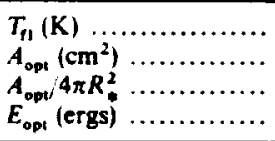 & $\begin{array}{c}9000 \\
1.1 \times 10^{13} \\
1.0 \times 10^{-4} \\
>4.6 \times 10^{33}\end{array}$ & $\begin{array}{l}9500 \\
5.6 \times 10^{17} \\
5.3 \times 10^{-3} \\
28 \times 10^{33}\end{array}$ & $\begin{array}{l}\cdots \\
\cdots \\
\cdots \\
\cdots\end{array}$ \\
\hline \multicolumn{4}{|c|}{ Coronal } \\
\hline 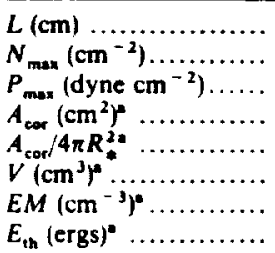 & $\begin{array}{l}3.8 \times 10^{10} \\
1.3 \times 10^{21} \\
180 \\
9.1 \times 10^{19} \\
0.0085 \\
7.1 \times 10^{30} \\
8.2 \times 10^{51} \\
1.9 \times 10^{33}\end{array}$ & $\begin{array}{c}<1.5 \times 10^{10} \\
<10 \times 10^{21} \\
<280 \\
1.9 \times 10^{19} \\
0.0018 \\
5.6 \times 10^{29} \\
2.5 \times 10^{51} \\
23 \times 10^{32}\end{array}$ & $\begin{array}{l}2.6 \times 10^{10} \\
1.5 \times 10^{21} \\
350 \\
1.7 \times 10^{21} \\
0.045 \\
8.8 \times 10^{31} \\
2.9 \times 10^{53} \\
4.6 \times 10^{34}\end{array}$ \\
\hline
\end{tabular}

- The coronal area coverage, volume, emission measure, and thermal energy listed here assume no correction for possible "dead spot "effects in the AD Leo observation. If a correction factor $f_{\infty}$ is applied, each quantity should be multiplied by $f_{d s}$. that the coroma is regarded as always being in a state of quasisteady hydrontatic equilibrium; (3) The pressure scale height $H_{P}$ is large compared to the loop semi-length $L$, so that the pressure in the loop is uniform, although changing with time; (4) The loop has a uniform cross-sectional area, which does not vary with time; (5) The conductive heat flux is given by the Spitzer formulation (Spitzer 1962); (6) Spatial variations of the flare heating rate in the corona can be averaged, i.e., the volumetric flare beating rate is assumed to be uniform in space (although it an vary in time); and (7) Radiative cooling takes place in the optically thin "coronal" approximation, with the temperature dependence of the cooling rate described by a single power-law fit over the temperature range of interest (see, e.g, the fit on p. 662 of Rosner, Tucker, \& Vaiana 1978, hereafter RTV, to the cooling law of Raymond \& Smith 1977).

Assumption (1) is true in the solar case and appears reasonable for stars Post facto, we find that assumptions (2) and (3) are satisfied by the flare evolution scenarios discussed in $\S 4.2$. The validity of assumption (4) is largely unknown, since there is virtually no observational constraint on how much the coronal loop area might change between the footpoint and summit. In the solar case, however, the observed loop area variations are small (Klimchuk et al. 1992). We have ignored possible area variations and assumed a uniform cross-sectional area. We note this as an uncertainty in our model calculations. Assumption (5) may not be satisfied in the earliest stages' of a flare, especially if the heating rate is very high and the initial coronal density is low (Fisher 1986). However, after one or two loop acoustic transit times, and after some of the initial evaporation has taken place, the increased coronal density will ensure that the heat flux approaches that given by the Spitzer formulation. The long rise times of our flares when compared to the loop acoustic transit time justify using the Spitzer formulation in our models. Assumption (6) is based on the high efficiency with which thermal conduction acts to smear out coronal temperature variations brought about by spatial variations in the flare heating rate. Further, Craig, McClymont \& Underwood (1978) have shown that the global properties of coronal loops (the pressure, apex temperature, etc.) are sensitive mainly to the total energy flux deposited in the corona and are insensitive to the placement of the energy deposition in the corona. Assumption (7) is reasonable for coronal loops at temperatures observed during flares, $T \gtrsim 10^{7}$ K (RTV).

With these assumptions, the flare loop energy equation for $\dot{P}$, the time rate of change of the loop pressure $P$, becomes

$$
\frac{3}{2} \boldsymbol{P}=Q-R-\frac{5}{2} P \frac{d v}{d z}-\frac{d F_{c}}{d z} .
$$

$F_{c}=-\kappa_{0} T^{s / 2} d T / d z$ is the conductive heat flux and $\kappa_{0}$ $\left(\sim 10^{-6}\right.$ in cgs units) is the Spitzer coefficient, $v$ is the plasma velocity in the enthalpy flux term, $Q$ is the volumetric flare heating rate, and $R$ is the optically thin cooling rate, assumed to be of the form $R=a n^{2} T^{\alpha}$. We take $\alpha=-\frac{1}{2}$ and $a=1.2 \times 10^{-19}$ (in cgs units) as reasonable approximations (see RTV) to more detailed calculations of coronal cooling rates (e.g. Raymond \& Smith 1977; Landini \& MonsignoriFossi 1990) for these temperatures. It is important to note that we have not considered the effects of possible differences of the elemental abundances (especially $\mathrm{Fe}$ ) from solar values. If it is determined that, e.g., Fe has a significantly different abundance on AD Leo than on the Sun, this could change the behavior of the plasma cooling function and the values of $a$ and $\alpha$ adopted 
here. In addition, there is strong evidence that the quiescent coronal abundances of Fe (and other low first-ionizationpotential elements) are enhanced from their photospheric values (see, e.g., Meyer 1991). It is not yet clear whether the flaring plasma in compact flare loops on the Sun shares this enhancement. In light of these complications, we have simply adopted solar photospheric abundances throughout our analysis.

The distance $z$ is measured along the loop from the chromospheric base, where the temperature $T$ assumes a chromospheric value $T_{\text {ch }}$; at the loop apex, a distance $L$ (i.e., the "loop length") away, the temperature attains its maximum value $T_{A}$, with $T_{A} / T_{\text {ch }} \gg 1$. The conductive flux vanishes at both $z=0$ (the chromosphere) and $z=L$ (the loop apex). We adopt an equation of state appropriate for fully ionized hydrogen, $P=2 n k T$, which relates the number density $n$ of hydrogen nuclei to $T$ and $P$ ( $k$ is the Boltzmann constant). To find the velocity $v$, we assume that the logarithmic rate of change of the density $(\dot{n} / n)$ along the loop is uniform and equal to the evaporation (or condensation) rate $\bar{N}$ divided by the total column number density $N$, of the loop. This is equivalent to assuming that the loop fills or drains homologousiy. The continuity equation can then be solved analytically for the velocity, resulting in

$$
v(z)=\dot{N} / n(z) \times\left(1-N(z) / N_{1}\right),
$$

where $N(z)=\int_{0}^{2} n\left(z^{\prime}\right) d z^{\prime}$, and $N_{1}=N(z=L) .^{3}$

If equation (4.1) is integrated from $z=0$ to $z=L$, the result is (see FH90)

$$
\frac{3}{2} \dot{P}=Q-\bar{R},
$$

where $Q$ and $\bar{R}$ denote loop-integrated spatial averages of $Q$ and $R(\bar{Q}=Q$, since $Q$ is assumed uniform). There are two limiting types of behavior, depeading on the relative amplitudes of $Q$ and $\bar{R}$ : If $Q \gg \bar{R}$, as one might expect early in a flare, then radiative losses are unimportant in determining the evolution of the loop, and the loop undergoes "strong evaporation," with the pressure in the loop increasing as the time integral of $Q$. On the other hand, if $\bar{R} \gg \boldsymbol{Q}$, as might happen late in a flare after much plasma has been evaporated and the heating rate has substantially decreased, then the loop pressure decreases in proportion to the average radiative cooling rate, resulting in "strong condensation." We moded the rise phase of flares in the strong evaporation limit and the decay phase in the strong condensation limit.

Note that if $\bar{R}=Q$, the loop is in energetic equilibrium and is therefore a "static" loop, obeying the loop scaling laws relating apex temperature, pressure, and loop length (RTV; Craig et al. 1978). The thermal structure and X-ray emissivity of static loops are discussed in detail in HF92 and in Hawley \& Fisher (1994). Static loop models are used to describe the flare

\footnotetext{
${ }^{3}$ Similar, but simplified, version of our energy equation, applied to the decay phase of flares on II Peg and Aled, were described by van den Oord \& Mewe (1989, hereafter VM) and Doyle van den Oord, \& Kellett (1992, hereafter DVK). In VM, an energy equation similar to eq. (4.1) was used. except that the enthalpy flux divergence tern dropped. in which case the energy equation results in exactly the same trmodynamic structure as that for a static loop. On the other hand. VM decount for variations in loop area. which our treatment negjects. In DVK, a order-of-magnitude solution to the continuity equation. plus an assumption of exponential decay of thermodynamic variables with time, were used to define a "constant velocity " cooling model for flare decay, which could the be compared with the observed decay of temperature and emission measure.
}

plasma at times between the rise and decay phases of flare EF1 (i.e., during the flare peak; see \$ 4.2).

\subsubsection{The Rise Phase}

The loss rate $\bar{R}$ is neglected during the rise phase, so that if evaporation continues throughout the rise time $\tau_{r}$, integration of equation (4.3) yields a pressure $P_{\max }$ given by $(3 / 2) P_{\max }=\int_{0}^{t}$ $Q(t) d t$. Here we identify $\tau$, with the elapsed time between the onset of the flare rise seen in the EUVE/DS count rate and the time of the peak count rate. Because the pressure at elapsed time $\tau_{r}$ depends only on the time integral of $Q$, we can replace the integral on the right-hand side by $Q_{2 v} \tau_{r}$, where $Q_{2 v}$ is the average value of $Q$ over the flare rise. Thus, the total energy deposited (per unit volume) in the loop $\left(\tau, Q_{\mathrm{av}}\right)$ is equal to the volumetric thermal energy at the end of rise phase, $(3 / 2) P_{\max }$.

We can now make use of analytical solutions for the temperature and density structure in a strongly evaporating loop (from Appendix A of FH90) to eliminate $Q_{2 v}$ in terms of the apex temperature $T_{A}$ and the loop semi-length $L: Q_{\mathrm{av}}=$ $(3 / 2) \zeta \kappa_{0} T_{A}^{7 / 2} / L^{2}$, where $\zeta=0.2913$. Results from Appendix $A$ also allow us to eliminate $P_{\max }$ in terms of $T_{A}, L$, and $N_{\max }$ (the column depth in one leg of the loop at the end of the rise phase), so that

$$
P_{\max }=2^{2 / 3} \eta 2 k T_{A} N_{\max } / L,
$$

where $\eta=0.4656$. After we make these substitutions, the solution to the time-integrated energy equation $\tau_{r} Q_{a v}=(3 / 2) P_{\max }$ reduces to

$$
N_{\max }=\frac{\zeta \kappa_{0} T_{A}^{S / 2} \tau_{r}}{2^{2 / 3} \eta 2 k L},
$$

which describes the amount of chromospheric plasma evaporated into the corona during the rise phase of the flare.

\subsubsection{The Decay Phase}

In the strong condensation limit, appropriate for the decay phase of a flare, equation (4.3) reduces to $(3 / 2) \dot{P}=-\bar{R}$. In $\S 3$ of FH90, we showed that a good approximation to the spatial average of the loss rate is

$$
\vec{R}=R N_{i}^{i} / L^{2},
$$

whwere $R / L^{2}$ is identical to the quantity $K$ defined in equation (13) of FH90, $N_{z}$ is the total coronal column depth in the loop, and $\lambda=14 /(7-2 \alpha)$. Recall that $\alpha=-0.5$ and thus $\lambda=1.75$. The value of $\hat{R}$ depends only on the physical constants $a, \alpha, k$, and $\kappa_{0}$, as defined in $\S 4.1 .1$. We relate the condensation rate through the loop footpoint $\dot{N}$, to $\dot{P}$ with the Ansatz

$$
\dot{N} / N_{\mathrm{r}}=\Omega \dot{P} / P \text {. }
$$

Results from a series of numerical gasdynamic simulations of flare loop decay (Jakimiec et al. 1992) suggest that $\Omega \sim 0.3$ during flare loop condensation. An interesting discussion of the "correct" value of $\Omega$ during flare loop decay can be found in Cargill, Mariska, \& Antiochos (1995). Adopting $\Omega=0.3$ and combining equations (4.3), (4.6), and (4.7) gives the single differential equation,

$$
\frac{d x}{d t}=-\frac{(2 / 3) \Omega \hat{K} N_{\max }^{\lambda}}{P_{\max } L^{2}} x^{\lambda+1-1 / \Omega} .
$$

Here $x=N_{t} / N_{\max }$, and $N_{\max }$ and $P_{\max }$ are the values of the column depth and pressure prior to the onset of strong condensation. If strong condensation occurs for a decay time $\tau_{d}$ after the loop has attained the column depth and pressure $N_{\max }$ 
and $P_{\max }$, integration of equation (4.8) then yiclds

$$
\frac{1-x_{d}^{1 / \Omega-\lambda}}{1 / \Omega-\lambda}=\frac{(2 / 3) \Omega K N_{\max }^{\lambda} \tau_{d}}{P_{\max } L^{2}},
$$

where $x_{d}$ is the value of $x$ after a time $\tau$, has elapsed.

We now seek to express $x_{d}$ in terms of an observed quantity, in this case the $E U V E / D S$ count rate. In principle, the observed EUV emission depends on both the temperature and emission measure (hence column depth or density) of the loop plasma. To understand these dependencies, we have computed theoretical $E U V E / D S$ count rates for a sequence of detailed loop models in the strong condensation limit (see Appendix B of FH90), with each loop model having a different apex temperature in the range from $1-3 \times 10^{7} \mathrm{~K}$, but with all models containing the same coronal column depth. We use the EUV plasma emissivities of Landini \& Monsignori-Fossi (1990) and the EUVE/DS detector response curve from CFAS. The emission in the $E U V E / D S$ passband is roughly the same for the entire sequence of models, indicating that it is relatively insensitive to apex temperature variations within this range. The emission scales as $N_{2}^{2}$, or equivalently, $x^{2}$. Therefore we can write $x_{d}=\left(C_{d} / C_{\max }\right)^{1 / 2}$, where $C_{\max }$ is the $E U V E / D S$ count rate at the flare peak, and $C_{d}$ is the count rate after a time $\tau_{d}$ has elapsed.

\subsubsection{Loop Lengths}

By approximating $P_{\max }$ and $N_{\max }$ in equation (4.9) with values of $P_{\max }$ and $N_{\max }$ achieved at the end of the rise phase, equations (4.5) and (4.9) can be combined to yield a single equation for the loop length $L$, which involves only $x_{d}, \tau_{d}, \tau_{p}$, physical constants, and $T_{A}$ (as estimated from the EUV spectroscopic data):

$$
L=\frac{1}{2^{2 / 3} 2 \eta k} \times\left[\frac{(2 / 3) R \Omega \tau_{d}\left(\zeta \kappa_{0} T_{A}^{5 / 2} \tau_{r}\right)^{\lambda-1}(1 / \Omega-\lambda)}{T_{A}\left(1-x_{d}^{1 / \Omega-\lambda}\right)}\right]^{1 / \lambda}
$$

Ignoring the $x_{d}$ dependence for the moment, and substituting $\lambda=7 / 4$ into the expression, equation (4.10) shows that $L \propto$ $\tau_{r}^{3 / 7} \tau_{d}^{4 / 7} T_{A}^{1 / 2}$. Thus, the loop length is relatively insensitive to $T_{A}$, so that modest uncertainties in the apex temperature of the loop have a small effect on derived values of $L$. Equation (4.10) also shows that long rise and decay times require large loop lengths. Once $L$ has been determined from equation (4.10), estimates for $N_{\max }$ and $P_{\max }$ can be obtained from equations (4.5) and (4.4). We emphasize that none of the quantities appearing in equation (4.10) require knowledge of the absolute flux in the DS detector; relative values of the count rate are sufficient to derive estimates of the loop length, coronal column depth, and loop pressure.

\subsection{Coronal Evolution During the EUV Flares on AD Leo}

From the EUVE/DS light curve for flare EF1 we obtain a rise time $\tau_{r} \sim 11 \mathrm{ks}$ (see Figs. 1 and $2 c$ and Table 2). The quiescent count rate observed before the flare is $\sim 0.27 \mathrm{~s}^{-1}$, while the count rate near the flare peak is $0.98 \mathrm{~s}^{-1}$. This implies a maximum flare-induced count rate of $\sim 0.71 \mathrm{~s}^{-i}$. Flare emission remains elevated with slight variations for nearly $4 \mathrm{hr}$ since the count rate during the peak is nearly constant, we assume that the coronal pressure and column depth maintain their maximum values during that time. The flare then decays rapidly for a time $\tau_{d} \sim 6 \mathrm{ks}$. After this, there is a continuing slow decay for most of the next day, but our models are only applicable during the initial rapid decay phase. At the end of that phase, the count rate has fallen to $54 \%$ of its peak value, thus, $x_{f}^{2} \sim 0.54$. From the EUV spectrum (Cully et al. 1995), we estimate the peak coronal temperature during flare EF1 to be $T_{A} \sim 25 \times 10^{7} \mathrm{~K}$. Given these parameters, we obtain from equation (4.10) a loop length $L \sim 3.8 \times 10^{10} \mathrm{~cm}$ (note this is $Z R_{\text {, }}$; from equation (4.5) we obtain a peak coronal column depth of $N_{\text {max }} \sim 1.3 \times 10^{21} \mathrm{~cm}^{-2}$; and from equation (4.4) we obtain a peak coronal pressure of $P_{\max } \sim 180$ dyne $\mathrm{cm}^{-2}$.

The quantities $L_{n} N_{\max }$, and $P_{\max }$ were estimated using only relative EUVE/DS count rates. If an absolute count rate is availab's, the loop cross-sectional area $A$, and hence the flare volume $\boldsymbol{V}$, the coronal emission measure $E M$, and the peak coronal thermal energy $E_{\text {th }}$ can also be determined by comparing the observed fux with that found from the loop models. We have computed the EUVE/DS flux expected at Earth during the flare peak using a static coronal loop with the values of $L$ and $T_{A}$ determined above, coronal emissivities taken from Landini \& Monsignori-Fossi (1990), the DS detector response curve taken from Figure 2 of CFAS, and estimates of the ISM opacity from Rumph, Bowyer, \& Vennes (1994) for an interstellar hydrogen column depth of $1 \times 10^{18} \mathrm{~cm}^{-2}$ (Paresce 1984), and a distance to AD Leo of 4.9 pc (Gliese \& Jahreiss 1991). The model calculations assume that none of the flare plasma radiation is absorbed by the star. We assume that the static loop model applies during the flare peak, sipce the count rate remains nearly constant for several hours. The average cross-sectional area of the loop is then simply the ratio of the observed count rate (counts $s^{-1}$ ) and the computed count rate (counts $s^{-1}$ ) for a loop of unit cross-sectional area.

For flare EF1, the loop cross-sectional area $A=9.1 \times 10^{19}$ $\mathrm{cm}^{2}$, which amounts to $0.85 \%$ of the stellar surface area if we adopt $R_{\varepsilon}=29 \times 10^{10} \mathrm{~cm}$ for AD Leo. (This radius was determined by first matching the measured spectral type of AD Leo to the corresponding effective temperature, then using the bolometric luminosity and effective temperature to find $R_{*}$ as in Pettersen 1980). The resultant flare loop volume is $V=2 L A=7.1 \times 10^{30} \mathrm{~cm}^{3}$, the peak emission measure is $\mathrm{EM}_{\max }=\left(N_{\operatorname{man}} / L\right)^{2} V=8.2 \times 10^{51} \mathrm{~cm}^{-3}$, and the peak thermal energy $E_{\mathrm{th}}=(3 / 2) P_{\operatorname{mex}} V=1.9 \times 10^{33}$ ergs. For EF 1 , $E_{\mathrm{ih}}$ is roughly 5 times the observed $E U V E / \mathrm{DS}$ energy $\left(E_{\mathrm{DS}}\right.$ in Table $2 E_{\mathrm{ch}}$ represents the total thermal energy contained in the evaporated plasma at the flare peak. In our model, the energy radiated during the strong condensation phase originates from this thermal reservoir.

Flare EF2 (Figs. 1 and $2 e$, Table 2) was both less intense and of shorter duration than flare EF 1 . The "quiescent" level for EF2 includes some postflare emission from EF1, resulting in an elevated prefiare count rate of $0.46 \mathrm{~s}^{-1}$. From the EUV spectrum of flare EF2 (Cully et al. 1995), we estimate a peak coronal apex temperature $T_{A} \approx 2 \times 10^{7} \mathrm{~K}$. The peak flare count rate was roughly $0.76 \mathrm{~s}^{-1}$, resulting in a flare-induced count rate of $0.30 \mathrm{~s}^{-1}$. The rise time for EF2 is poorly determined, and we can provide only an upper limit, since the count rate reaches its peak value only one orbital data gap after being at the quiescent level, i.e., $\tau_{\text {r }} \$ 6 \mathrm{ks}$. The decay timescale is also poorly determined for the same reason, namely, the count rate returns to its preflare value one orbital gap after being near its maximum. Thus we can set only an upper limit $\tau_{d} \leqq 6 \mathrm{ks}$. Because there are no reliable measurements for the count rate during the decay phase, we can say only that $x_{d}^{2} \geq 0$ after $6 \mathrm{ks}$. With generous allowance for the uncertainties, we use these limits to estimate $L, N_{\max }$, and $P_{\max }$ from our models. We find 
$L \sim 1.5 \times 10^{10} \mathrm{~cm}, N_{\max } \sim 1.0 \times 10^{21} \mathrm{~cm}^{-2}$, and $P_{\max } \sim 280$ dyne $\mathrm{cm}^{-2}$. The estimates are essentially unconstrained but are physically reasonable. Matching a detailed static loop model with these parameters to a flare-induced count rate of $0.30 \mathrm{~s}^{-1}$ yields a loop cross-sectional area $A=1.9 \times 10^{19} \mathrm{~cm}^{2}$ (or $0.18 \%$ of the stellar surface area), a flare volume $V=5.6 \times 10^{29} \mathrm{~cm}^{3}$, a coronal emission measure $E M=2.5 \times 10^{51} \mathrm{~cm}^{-3}$, and a peak thermal energy $E_{\mathrm{th}}=2.4$ $\times 10^{32}$ ergs, or about 4 times the observed EUVE/DS energy in Table 2.

The derived loop parameters for flares EF1 and EF2 are summarized in Table 3.

\subsection{Coronal Evolution of the $A U$ Mic Flare}

We can apply the same analysis used to determine the AD Leo flaring coronal parameters to the flare peak behavior in the giant $A U$ Mic flare observed by EUVE and reported in Cully et al. (1993). The distance to AU Mic is 9.3 pc (Gliese \& Jahreiss 1991) and we estimate the stellar radius, using the same method as for AD Leo, to be $R_{*} \simeq 5.5 \times 10^{10} \mathrm{~cm}$. The interstellar column depth of $2 \times 10^{18} \mathrm{~cm}^{-2}$ is again taken from Paresce (1984), from which the EUV extinction is computed using Rumph et al. (1994). Cully et al. (1993) estimated the

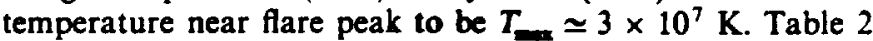
gives our values for the measured rise and decay timescales and the corresponding count rates. Note that our analysis applies only to the rapid rise and decay seen during the first large peak between 197.46 days-197.60 days (see Fig. 2 of Cully et al. 1993). Using the published count rates, we find a coronal loop length $L=2.6 \times 10^{10} \mathrm{~cm}$, a peak pressure $P_{\max }=350$ dyne $\mathrm{cm}^{-2}$, and a maximum column depth of $N_{\max }^{\max }=1.5 \times 10^{21}$ $\mathrm{cm}^{-2}$. Using the observed count rates, we find a coronal loop cross-sectional area of $4.5 \%$ of the stellar surface area, which results in a coronal area coverage of $1.7 \times 10^{21} \mathrm{~cm}^{2}$, an emission measure of $2.9 \times 10^{53} \mathrm{~cm}^{-3}$, and a peak thermal energy of $4.6 \times 10^{34} \mathrm{ergs}$. These values are included in Table 3. Evidently the AU Mic flare was much more energetic than the largest AD Leo flare (EF1) although the flaring loop (or loop arcade) was more compact. We also found that for AU Mic, a static loop model with the derived loop length had too large a loop pressure compared to the value found from equation (4.4). A "strongly evaporating "loop, on the other hand, gives a consistent pressure and was used to determine the quoted crosssectional area and volume. A strongly evaporating loop is also consistent with the rapid rise to the peak count rate during the AU Mic flare, as compared to flare EF1 (which rose more slowly and stayed at the flare peak value for considerably longer, and was best fit by a static loopl

\subsection{Loop Sizes and Flare Geometry}

The coronal loop lengths and areas we find for flares EFI and EF2 (Table 3) are considerably larger than those determined for large solar flares, in which a flare loop might have a length of a few $\times 10^{9} \mathrm{~cm}$ and a total area of less than $10^{-4}$ of the solar surface. This qualitative difference between solar and stellar flare loops is well known, although our loop lengths are somewhat longer than estimates from earlier studies $(L \sim 1-$ $\left.2 \times 10^{10} \mathrm{~cm}\right)$. Because our technique for estimating loop lengths and cross-sectional areas is new, we now compare and contrast our methods with those obtained previously. We also comment on other interpretations of flare decay that do not involve loop condensation.

Most previous estimates for the sizes of flaring coronal loops on dMe stars have been possible only when high-energy (usually soft $X$-ray) data were available, as in Haisch et al. (1987), Doyle et al. (1988), and Tagliaferri, Doyle, \& Giommi (1990). The method used by these authors involves first determining the emission measure of the flare from the total measured luminosity coupled with an estimate of the emissivity (based on an assumed or measured temperature plus an adopted cooling law). Next, the mean density in the flare plasma is derived by equating the measured flare decay time to the radiative cooling rate (again assuming a cooling law), at fixed temperature. With the emission measure and density established, the volume is obtained. To derive a loop length, an aspect ratio between the loop diameter and the loop length is assumed, and then the measured flare volume can be related to the loop length. Typically, solar values of the aspect ratio $(0.1-$ $0.2)$ are invoked, resulting in loop lengths of $1-2 \times 10^{10} \mathrm{~cm}$.

Applying this analysis to our data for EF1 results in a loop length of $4 \times 10^{10} \mathrm{~cm}$ (similar to our model result in Table 3), a mean density of $9 \times 10^{10}$ (about 2.5 times greater than our value), and hence a smaller area coverage. The reason for the difference in the density is that our condensation model accounts self-consistently for changes in both the temperature and density as the flare evolves.

In contrast to this earlier work, our estimate of the loop length (eq. [4.10]) depends only on measured timescales and the coronal temperature and is determined independent' $y$ of the volume emission measure. Because of this, the emission measure can be used to find the flare volume and the total loop cross-sectional area. In our analysis, no assumptions about the loop aspect ratio are necessary. Rather, the total crosssectional area is a product of our analysis. If one chooses to assume that ail loops have a solar aspect ratio of $0.1-0.2$, the total cross-sectional area from our analysis can then be used to find the number of such loops that comprise a flaring coronal arcade. If taken as single loops, the aspect ratios for flares EF1 and EF 2 would be 0.15 and 0.18 , respectively. We emphasize again that equation (4.10) shows that a flare with a long rise and decay time must take place within a long loop or within an arcade of long loops.

Our technique for estimating the loop length is most similar to the method described by Reale et al. (1988). These authors computed a series of $\mathrm{X}$-ray light curves from several different flare loop hydrodynamic simulations, each assuming a differing loop length. These light curves were then compared to the observed light curve from the 1980 August 20 flare observed on Proxima Centauri by the Einstein satellite. The energy input phase in their simulations was determined by the observed rise phase in the flare; the behavior of the decay phase was then determined by the assumed loop length. They found that if the loop length was too long, the computed decay was slower than that observed; if it was too short, the computed decay was too fast. This technique for finding $L$ is basically the same as ours. except that we have exploited semianalytic models for the flare rise and decay phases, rather than employing detailed hydrodynamic simulations.

Previous work on the active pre-main-sequence dwarf $A B$ Dor (Collier-Cameron, \& Robinson 1989) suggested that centripetal acceleration was required to support the very large loops inferrod from their data. The loop lengths we find for AD Leo are not as large; coupled with the higher gravity and slower rotation speeds of $A D$ Leo and $A U$ Mic, we find that centripetal forces are not important for the flare loops in our model. 


\subsection{Alternatives to Simple Exaporation and Condensation in Loops}

If the flare rise and decay phases are a result of different physical scenarios than the ones we have assumed in this paper, then the interpretation of the EUVE/DS light curve evolution will differ accordingly. Two specific alternatives to simple loop evaporation and condensation deserve comment, namely, reconnection in two-ribbon loop arcades (Poletto, Pallavicini, \& Kopp 1988, hereafter PPK), and modeling the flare decay as the ejection of a coronal loop or plasmoid (CFAS). In the former case, PPK fitted the decay phases of two stellar flares (the 1985 August 6 flare on EQ Peg observed by EXOSAT, and the 1980 August 20 flare on Prox Cen observed by the Einstein satellite) with the coronal arcade reconnection model of Kopp \& Poletto (1984, hereafter KP). In that model, magnetic field lines that have been blown open early in the flare reconnect into closed field lines As time proceeds, a sequence of closed coronal loop arcades is formed, in which the most recently formed arcade encloses those formed earlier. PPK assume that the reconnection energy release rate predicted by KP can be matched in time with the observed $X$-ray light curves. $A$ form for the temporal variation of the reconnection rate in the model calculations must be assumed, but this choice can be adjusted in such a way as to obtain good agreement between the theory and the observations.

The direct comparison between the theoretical model and the observations in PPK glosses over several important issues. Assuming that the energy release rate and the $X$-ray luminosity track in phase with each other is tantamount to assuming that the corona can respond instantaneously via evaporation and condensation to the heat deposited in the reconnected loops. We have shown in $\$ 4.1$ that this is not the case. If coronal evolution is governed by the KP reconnection model, then the response of each one of the coronal loops (or equivalently, each arcade) is governed by equations similar to those we discussed in $\$ 4.1$. Evolution of the entire active region with time would be determined by a convolution between the energy release rate due to reconnection and the $\mathrm{X}$-ray response function for each set of loop arcades. Using a solar example as a guide to what might occur during a large stellar flare, consider the 1980 May 21 two-ribbon fiare observed by SMM (Antonucci, Gabriel, \& Dennis 1984). Even though the decay phase does appear to involve the reconnection of open field lines into nested sequences of closed coronal loops (see Poletto \& Kopp 1985,1988 ), we have found that a single-loop model adequately describes the evolution of the brightest flare plasma, at least through the early part of the decay phase (Fisher \& Hawley 1990). We believe this is because near the peak of the flare, the brightest loop(s) dominate the emission from the entire active region, and these loops have only one particular length scale.

An alternative flare decay scenario has been suggested by CFAS in their study of the EUVE/DS light curve of the 1992 July 15 flare observed on the dMe star AU Mic. This flare exhibited a brief, intense period of emission (which we discussed in $\S 4.3$ ), lasting roughly $2 \mathrm{hr}$, followed by a much longer, slow decay phase lasting roughly $1-2$ days. The two different timescales suggest that two different phenomena occurred during that flare. For the slow decay phase, CFAS propose a model that is based on the ejection of bright coronal loops or plasmoids from the star, accompanied by their expansion into the interstellar medium. While this mechanism could explain very long flare decay times, it cannot cope with the shorter decry timescales of flares EF1 and EF2 on AD Leo (or the rapid dowy phase of the AU Mic flare), because the required expmion velocities for the ejected loops would be unphysically large, and/or the initial densities would be sufficiently large that radiative cooling would dominate expansion cooling, violating one of the precepts of their model. Therefore we believe that the initial flare decay seen during the 1992 July I5 AU Mic fare, as well as the decay phases of flares EF 1 and EF2, are casoed by cooling and condensation within closed coronal loops.

\section{DISCUSSION}

Analysis of the optical data alone allowed us to place the observed AD Leo flares in the context of previous optical flare data, with the result that they are unusual in terms of longevity and relatively small enhancement but large total energy, though they are by no means extraordinary. The optical continuum and line emission evolve together in typical fashion, and a blackbody fit to the continuum gives temperatures and area covera $=$ similar to other flares. The EUVE/DS data, together with our flare models, give an improved determination of the coronal loop parameters and flare geometry. By combining in ormation from the optical and $E U V E / D S$ data sets, we now conatruct a picture of flare EF1 as a whole and compare it with what is known about solar flares.

\subsection{A Picture of Flare EFI}

,

We adopt a coronal loop geometry with footpoints in the photosphere and height $\sim 1 R_{*}$ above the stellar surface. We refer to the "loop" as a single entity but do not exclude an arcade of loope of similar length whose total area combines to give the inferred area coverage.

The flare is firs seen as a rapid increase in the optical continuum, observed in the $U$-band photometry. Hudson et al. (1992) showed that white-light and hard $\mathrm{X}$-rays in solar flares were coincident both in space and time. Since the hard $X$-rays are emitted by accelerated nonthermal electrons, we identify the optical contioum radiation with the impact sites where the electrons are depositing their energy via collisions in the dense lower atmosphere. Our analysis of the optical emission indicates that the fractional area coverage $(\sim 0.01 \%$ of the stellar area) is much smaller than the estimated coronal crosssectional area $(\sim 1 \%)$. On the Sun, the chromospheric flare kernels imaged in $\mathrm{H} \alpha$ or hard $\mathrm{X}$-rays are also found to be much smaller in exteat than the coronal flare loop areas (Canfield et al. 1991). One possible explanation for these differences in area coverage is the convergence of magnetic field lines in the chromosphere and photosphere, which results from the transition between a space-filling potential or force-free field in the corona, to thin, discrete magnetic flux tubes confined by gas pressure at the photosphere.

Since EFl occurred in a rather long loop, the initial flare heating seen in the $U$ band did not produce an immediate change in the coronal emission measure, although evaporation was probably beginning at this time.

The second stage of the flare is characterized by a plateau in the $U$-band cmission which we identify with a period of continued electron precipitation possibly at fresh impact sites within the loop. The loop is undergoing strong evaporation and the coronal emission begins to increase.

At the end of this period, the $U$-band emission again rises sharply to a second peak, while the coronal emission reaches its peak. The combination of optical and $E U V E / D S$ data over 
the entire flare to this time (i.e., the time of maximum coronal emission measure), now allows us to examine the evidence for a stellar Neupert effect. As discussed in $\S 1$, the canonical solar model leads to the prediction that hard X-rays and white-light emission will precede the soft $\mathrm{X}$-ray (EUVE/DS) emission and, in fact, the $E U V E / D S$ emission should scale with the time integral of the white-light energy. The time delay we observe between the optical flare and the coronal emission could therefore be interpreted as a stellar Neupert effect. In Figure 3 we show the time-integrated $U$-band energy superposed on the observed $E U V E / D S$ light curve (with the count rate expressed in energy units). The temporal behavior matches well and, if anything, shows that the nonthermal electron power (which must be at least as great as the power observed in $U$ ) is more than adequate to produce the observed EUV (i.e., coronal) emission during the flare evolution. In $\$ 5.3$ we discuss some previously observed flares and find that they also appear to show a Neupert effect. Evidently, the same underlying heating processes, direct excitation by accelerated electrons and evaporation of chromospheric material into the corona, can account for the temporal relationship between coronal and optical continuum emission on both dMe stars and the Sun. However, stellar flares can be far more energetic and exhibit heating timescales that are significantly longer than those observed in the solar case. We discuss some possibilities for why these differences might arise in $\S 5.2$ below.

Following the rise phase, the coronal emission reaches a plateau near its maximum value for a period of several hours. The radiative cooling time in the corona during this period is about $10^{4} \mathrm{~s}$; since the emission shows no decay over that timescale, we surmise that there must be continued heating. The heating is not accompanied by evaporation, since the emission measure does not increase. One possibility may be that the flare heating mechanism which formerly reached the lower atmosphere is now depositing its energy directly into the corona; we address this further in $\$ 5.2$. Unfortunately, we have no optical observations to confirm the lack of lower atmospheric heating during this phase.

We note that the poor time resolution of the EUV data allows an alternative explanation for the emission during this time period. It is possible that the "flare peak" between $\sim 36$ $40 \mathrm{hr}$ is actually a composite of emission from two flares, one of

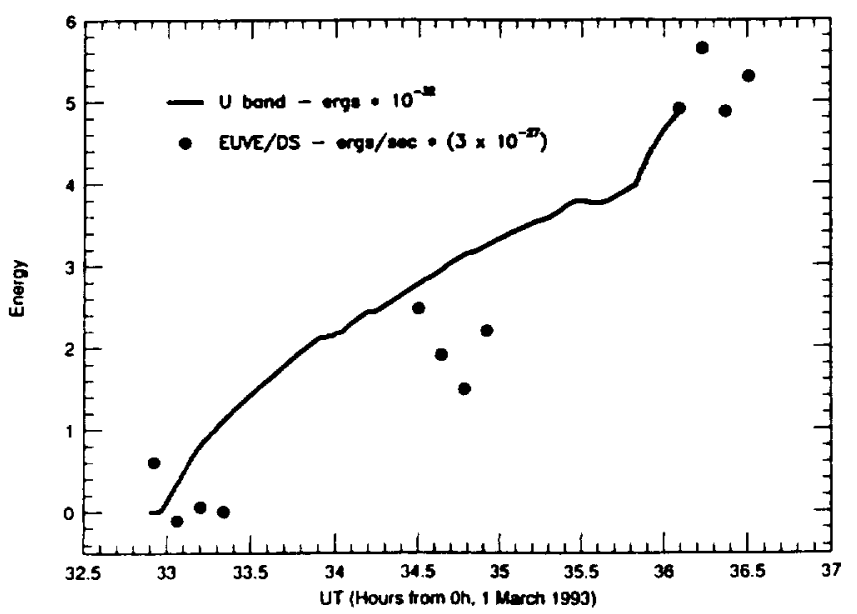

FIG. 3.-Solid curve shows a time integral of the $U$-band luminosity (with the preflare luminosity subtracted) during the rise phase of flare EFI. Filled circles show the EUVE/DS count rate during this period, converted to luminosity units. We argue that the behavior shown here provides evidence for a stellar Neupert effect. which peaks at $\sim 36 \mathrm{hr}$, starts to decay in the next orbit, and then is joined by a second flare which peaks at $\sim 40 \mathrm{hr}$. This would remove the need for a continuous heating process lasting some $4 \mathrm{hr}$ at the flare peak. Our data do not allow us to distinguish between this two-flare scenario and a single-flare plateau phase. However, if we assume a plateau, our analysis of the loop during this phase shows that a static model is consistent with the loop length and pressure derived from equations (4.10) and (4.4), which does support a slow deposition of heat directly into the corona.

The coronal emission next enters a phase of relatively rapid decay, which we identify as the strong condensation phase. Flare heating has ceased, and the coronal plasma cools and condenses down the loop into the chromosphere. It is the combination of the rise and decay phase timescales, together with our theory of evaporating and condensing loops, which allows us to determine the loop length unambiguously. Thus, the long loop length we derive follows directly from the observed long rise and decay times. As mentioned earlier, this does not preclude a scenario in which several episodes of heating take place within an arcade of loops of this characteristic length.

It is possible that an ensemble of short loops (which would respond more rapidly to flare heating), together with a contrived heating profile, could mimic the natural response of a long loop. We regard this as an unnecessary complication unless further observational constraints on the loop length are discovered.

Finally, the coronal emission switches from a (relatively) rapid decay to an extended slow decay, lasting most of the next day. Our models do not address this phase. It may represent a relaxation process as the loop returns to quiescence, or, as suggested in CFAS for AU Mic, it may be the emission from an expanding and cooling coronal mass ejection. One intriguing feature of the slow decay phase on both AU Mic and AD Leo is the presence of a second small flare (here, EF2) which occurred approximately 1 day after the initial large flare (here, EF1). This may be merely coincidence, though Brown et al. (1994) also report a similar secondary flare on HR 1099 approximately 1 day after a large flare. Further observations are required to test whether the second flare is a typical (and perhaps important) feature of stellar flares.

\subsection{Impulsive Phase Quenching}

As discussed in $\$ 4$, the thermal response times and the amounts of energy which can be stored in hot coronal plasma should depend in a predictable way upon the length of coronal loops. We showed, in particular, that the loop length $L \propto$ $\tau_{f}^{3 / 7} \tau_{d}^{4 / 7}$. A more subtle issue is whether the coronal loop length and/or cross-sectional area can have a direct effect on the duration of the impulsive phase of flares. Such a relationship is suggested by the longer duration of impulsive optical continuum emission in many stellar flares as compared to solar flares. One possibility is that impulsive phase timescales are set by an intrinsic flare reconnection rate and the amount of magnetic flux available to be reconnected. If this were the case, we might expect the duration of the impulsive phase to be related to the coronal loop cross-sectional area or diameter, which is often found to be larger in the stellar case than in the solar case. Another possibility, described below, is that the impulsive phase of flares ends when it is "quenched" by a high coronal density. The time it takes for the quenching to occur is a strongly increasing function of loop length.

The impulsive phase acceleration mechanism, whatever it is, must obey at least two constraints: (1) Energy is extracted (by 
some means) from nonpotential magnetic fields and converted into the kinetic energy of the accelerated electrons, and (2) the acceleration mechanism must be effective enough to overcome Coulomb losses from particles in the ambient plasma. An extended period of flare energy release will result in an increasing level of the overall coronal density, because of chromospheric evaporation. If the impulsive phase is to persist, the acceleration mechanism must continue to operate, even in the face of the increased Coulomb losses. We suggest that the impulsive phase will be quenched when the ambient coronal density reaches a critical threshold $\boldsymbol{n}_{\text {erh }}$, where Coulomb losses overcome acceleration. The time it takes for this to occur would then determine the duration of the impulsive phase. Because the loop length $L$ affects how quickly coronal loops can fill with evaporated plasma, we then expect a relationship between coronal loop length and the duration of the impulsive phase. For a given flare apex temperature $T_{A}$, the quantity $\dot{n}=\dot{N}_{1} d / L$ scales as $L^{-2}$ (eqs. [A8] and [A9] of FH90) in the strong evaporation regime. Writing the duration of the impulsive phase $\tau_{\mathrm{Imp}} \simeq n_{\text {criv }} / \dot{n}$ gives $\tau_{\ldots} \propto L^{2}$, meaning that the impulsive phase for stellar flares occurring in long loops should indeed be much longer than that for solar flares.

Thus we have identified at least two possibilities for why the duration of the impulsive phase should depend on loop length or cross-sectional area, and hence why the impulsive phase for many stellar flares might last longer than that for solar flares. However, determining which (if either) scenario is operable can probably only be addressed by examining existing data sets of solar flares, where both good spatial and temporal resolution are available.

The wide range of observed stellar rise times, and the varying degree of correlation between optical and high-energy light curves that have been cited in previous stellar flares (see $\$ 1$ ), thus finds a natural explanation in terms of a wide variation of loop sizes in different stellar fiares.

\subsection{Comparison with Previous Flare Observations}

Several previous flare observations show evidence for a stellar Neupert effect such as the one reported here. Examination of Figures 2 and 3 from Kahler et al. (1982) shows that the $U$-band emission peaks during a time when the $X$-ray count rate is rising rapidly for two separate flares on YZ CMi. A similar result is visible in Figure 11 of de Jager et al. (1989), during a flare on UV Ceti.

The existence of a physical connection between the coronal and chromospheric emission, as manifested by the Neupert effect, provides a straightforward explanation for the empirical correlations between energy emitted in X-rays, chromospheric emission lines, and optical continuum emission discussed in Butler et al. (1988), Haisch (1989), and HP91. The energy correlations extend over several orders of magnitude in total flare energy and apply to both the Sun and to flare stars. HP91 interpreted this result as evidence for a constant heating rate in flares of very different total energy, so that the energy difference depended on the area coverage and duration of the heating. Our new data and models suggest that the primary source for energy deposition observed in solar flares, i.e., a flux of nonthermal electrons, can also produce the emission seen in stellar fares, provided that the flare occurs in a loop of appropriate length as determined by the rise and decay timescales. It is then the relative efficiency of the nonthermal electrons in heating the chromospheric material directly to produce the optical emission, and in evaporating it to coronal temperatures to produce the soft $\mathrm{X}$-ray emission, that must remain nearly con- stam in order to obtain the observed empirical correlation between the energies.

Evidence for such a constant relative efficiency is indeed suggested in the case of solar fiares by the work of Kane \& Donnelly (1971), Donnelly \& Kane (1978), and Kane, Frost, \& Donnelly (1979), who showed that the ratio of long-wavelength EUV radiation (emitted primarily from the transition region and chromosphere) to hard $X$-rays was nearly constant over a very wide range of solar energies. A more recent study of whitelight emixsion and hard X-rays (Neidig \& Kane 1993) showed similnr results.

\section{CONCLUSIONS}

Our simultaneous optical and $E U V E / D S$ data obtained during a flare on AD Leo provide strong evidence for a stellar Neupert effect in which the $E U V E / D S$ light curve is roughly proportional to a time integral of the $U$-band light curve. When we examine earlier simultaneous optical and soft X-ray stellar flare data, we find confirmation of this phenomenon. The well-established spatial and temporal correlation of whitelight emission and hard X-ray emission in solar flares, together with the identification of the hard $X$-ray emission as a signature of nonthermal bremsstrahlung from a flux of energetic electrons, suggests that the optical continuum produced in stellar flares is a real observational signature of impulsive phase nonthermal electron heating. Therefore, the model that has been developed to explain solar fiare observafions is equally applicable to stellar flares. Differences in rise and decay timescales, and fiare energetics, can be ascribed to differences in the sibes of the coronal loop structures.

We acknowledge interesting and useful discussions with Brendan Byrne, Dick Canfield, Hugh Hudson, David Lambert, Mihalis Mathioudakis, Bob Stern, and Ossy Siegmund. We wish to thank the staffs of the Center for EUV Astrophysics, the Lict Observatory of the University of California, the McDonald Observatory of the University of Texas, and the Nordic Optical Telescope for their assistance in the acquisition of the data presented here. We also thank Peter Vedder and Jeremy Drake for kindly providing us with observing time on the $\mathbf{4 0}$ inch telescope at Lick Observatory, and the directors of Lick and McDonald Observatories for giving us discretionary time on the 24 inch and CAT telescopes at Lick and the 36 inch telescope at McDonald, on very short notice. This research was supported in part by NASA grant NAG 5-2275 to the University of Hawaii and by NSF Young Investigator award AST 94-57455 to Michigan State University. S. L. H. was partially supported by a Hubble fellowship through NASA Grant No. HF-1017.01-91A awarded to the Space Telescope Science Institute which is operated by the Association of Universities for Research in Astronomy, Inc., for NASA under contract No. NAS 5-26555. Part of the research was done at the Lawrence Livermore National Laboratory under the auspices of the United States Department of Energy, through contract no. W-7405-ENG-48. G. H. F. was supported by NASA grant NAGW-3429, and NSF grant AST 92-18085. S. L. C. acknowledges support by the NASA GSRP.

It is with great pleasure that we dedicate this paper to Frank Q. Orrall, on the occasion of his retirement from the University of Hawaii. It has been our good fortune to know Frank as friend and colleague, and it is especially fitting that we are able here, in a paper on stellar coronal flares, to acknowledge his many contributions to solar coronal physics over the course of a long and distinguished career. 
REFERENCES

Abbott, M. J., Miller, A. \& Gavin, L. 1993, EUVE Guest Observer Center Software User's Guide (Berkeley: Center for EUV Astrophysics)

Antonucci E Gabriel, A. H, \& Dennis, B. R. 1984, ApJ, 287,917

Basri, G. Wilcots, E., \& Stout, N. 1989, PASP, 101, 528

Bastian, T. S. 1990, Sol. Phys., 130, 265

Bookbinder, J. A. Waiter, F. M., \& Brown, A. 1992, in ASP Conf. Proc. 26, Cool Stars, Stellar Systems and the Sun, od. M. Giampapa \& J. Bookbinder (San Francisco: ASP), 26, 27

Brown, A., Skinner, S., Stewart, R., Drake, S, \& Deeney, B. 1994, BAAS, 26, 1463

Butler, C. J., Rodono, M. \& Foing B. H. 1988, A\&A, 206, LI

Byme, P. B. 1979 , MNRAS, 187, 153

Canfield R. C. Zarro, D. M., Wülser, J.-P., \& Dennis, B. R. 1991, ApJ, 367, 671

Canfield, R. C., et al. 1986, in Energetic Phenomena on the Sun, ed. M. Kundu

\& B. Woodgate (NASA Conf. Publ. 2439), chapter 3

Cargill, P. J., Mariska, J. T., \& Antiochos, S. K. 1995, ApJ, 439, 1034

Coltier-Cameron, A., \& Robinson, R. D. 1989, MNRAS, 236, 57

Craig, I. J. D., McClymont, A. N. \& Underwood, J. H. 1978, A\&A, 70, 1

Cristaldi, S., Gershberg, R. E., \& Rodono, 1980, A\&A, 89, 123

Cully, S. L., Fisher, G. H., Abbott, M. J., \& Siegmund, O. H. W. 1994, ApJ, 435, 449 (CFAS)

Cully, S. L., Siegmund, O. H. W., Vedder, P. W. \& Vallerga, J. V. 1993, ApJ, 414, L49

Cully, S. L., et al. 1995, in preparation

de Jager $C$ et al 1989 , A\&A, 211, 157

Dennis, B. R \& Schwartz R. A. 1989, Sol. Phys., 121, 75

Dennis, B. R. \& Zarro, D. M. 1993, Sol. Phys., 146, 177

Donnelly, R. F., \& Kane, S. R. 1978, ApJ, 222, 1043

Doyle, J. G., Butler, C. J., Byrne, P. B., \& van den Oord, G. H. J. 1988, A\&A, 193,229

Doyle, J. G., van den Oord, G. H. J., \& Kellett, B. J. 1992, A\&A, 262, 533 (DVK)

Fisher, G. H. 1986, in Lecture Notes in Physics, Vol. 255, Radiation Hydrodynamics in Stars and Compact Objects, ed. D. Mihalas \& K.-H. Winkler (Berlin: Springer), 53

Fisher, G. H. \& Hawley, S. L. 1990, ApJ, 357, 243 (FH90)

Giampapa, M. S. 1983, in Activity in Red Dwarf Stars, ed. P. B. Byrne \& M. Rodono (Dordrecht: Reidel), 223

Giampapa, M. S., Africano, J. L., Klimke, A., Parks, J., Quiqley, R. J., Robinson, R. D. \& Worden. S. P. 1982, ApJ, 252, L39

Gliese, W. \& Jahreiss, H. 1991, Third Catalogue of Nearby Stars, preprint

Grinin, V. P. 1983, in Activity in Red Dwarf Stars, ed. P. B. Byrne \& M. Rodono (Dordrecht : Reidel), 613

Gurzadyin. G. A. 1988, ApJ, 332, 183

Hawiey, S. L \& Pettersen, B. R. 1991, ApJ, 378, 725 (HP91)

Hawley, S. L., \& Fisher, G. H. 1992, ApJS, 78, 565 (HF92)

1994, ApJ, 426, 387

Haisch. B. M. et al. 1981, ApJ, 245, 1009

Haisch, B. Antiochos, S. K., Golub, L.. \& Vaiana, G. S. 1983, ApJ, 267, 280

Haisch. B. M.. Butler, C. J., Doyle, J. G., \& Rodono, M. 1987, A\&A. 181, 96

Haisch, B. M. 1989, A\&A. 219, 317

Henoux, J-C., Aboudarham, J., Brown, J. C., van den Oord, G. H. J., van Driel-Gesztelyi, L.. \& Gerlei, O. 1990, A\&A, 233, 577
Houdebine E R \& Doyle J G 1994a, A\&A, 289, 169 $1994 \mathrm{~b}, \mathrm{~A} \& \mathrm{~A}, 289,185$

Houdebine, E. R., \& Panagi, P. M. 1990, A\&A. 231, 459

Hudson, H. S Acton, L. W., Hirayama, T., \& Uchida. Y. 1992. PASJ, 44, L77

Jakimiec, J., Sylwester, B., Sylwester, J., Serio, S., Peres, G., \& Reale, F. 1992. A\&A, 253, 269

Johnson, H. L. 1966, ARA\&A, 4,93

Kahler, S., et al. 1982, ApJ, 252, 239

Kane, S. R \& Donnelly, R. F.1971, ApJ, 164, 151

Kane, S. R., Frost. K. J., \& Donnelly, R. F. 1979, ApJ, 234, 669

Klimchuk, J. A., et al. 1992, PASJ, 44, L 181

Kopp, R. A., \& Poletto, G. 1984, Sol. Phys., 93, 251 (KP)

Landini, M. \& Monsignori-Fossi, B. C. 1990, A\&AS, 82, 229

Landolt, A. U. 1973. AJ, 78, 959 1992 AJ, 104, 340

Lin, R. P., \& Hudson, H. S. 1976, Sol. Phys., 50, 153

Malina, R. F. \& Bowyer, S. 1991, in Extreme Ultraviolet Astronomy, ed. R. F. Malina \& S. Bowyer (New York: Pergamon), 397

Massey, P. Strobel, K., Barnes, J. V., \& Anderson. E. 1988, ApJ, 328, 315

McCarthy, J. K., Sandiford, B. A., Boyd, D., \& Booth, J. 1993, PASP. 105, 881

Meyer, J.-P. 1991, Adv. Space Res., $11(1), 269$

Miller, A. 1994, private communication

Mochnacki, S, \& Zirin, H. 1980, ApJ, 239, L27

Moffett, T. J. 1974, ApJS, 29, 1

Moffett, T. J., \& Bopp, B. W. 1976, ApJS, 31,61

Neidig, D. F., \& Kane, S. R. 1993, Sol. Phys., 143, 201

Neupert, W. M. 1968, ApJ, 153, L59

Paresce, F. 1984, AJ, 89, 1022

Pettersen, B. R. 1980, A\&A, 82,53

Pettersen, B. R. Coleman, L. A., \& Evans, D. S. 1984, ApJS, 54, 375

Pettersen, B. R. Panov, K. P., Sandmann, W. H., \& Ivanova, M. S. 1986 A\&AS, 66, 235

Poletto G. \& Kopp, R. A. 1985, in Lower Atmosphere of Solar Flares. Relationships Between Low Temperature Plasmas and High Energy Emissions ed. D. F. Neidig (Sunspot, NM: National Solar Observatory. Sacramento Peak) 353

1988, Sol. Phys., 116, 163

Poletto, G. Pallavicini, R \& Kopp, R A 1988. A\&A, 201, 93 (PPK)

Raymond, J. C., \& Smith, B. W. 1977, ApJS, 35, 419

Reale, F., Peres, G., Serio, S., Rosner, R., \& Schmitt, J. H. M. M. 1988, ApJ. 328,256

Rodono, M et al 1989, in IAU Collog, 104, Spec. Publ. Catania Astrophys Obs., ed. B. M. Haisch \& M. Rodono, p. 53

Rosner, R., Tucker, W. H., \& Vaiana, G. S. 1978, ApJ, 220, 643 (RTV)

Rumph, T., Bowyer, S., \& Vennes, S. 1994, AJ, 107, 2108

Siegmund O H. W et al 1986, in Proc. SPIE Instrumentation in Astronomy VI ed. D. Crawford (Bellingham. WA: SPIE), 627,660

Spitzer, L. 1962, The Physics of Fully Ionized Gases, (2d ed.; New York Interscience)

Tagliaferri, G., Doyle J. G., \& Giommi, P. 1990, A\&A, 231, 131

Valenti, J 1994, Ph. D dissertation in Astronomy, Univ. California, Berkeley van den Oord G. H. J \& Mewe, R. 1989, A\&A, 213, 245(VM)

vogt, S. S. 1987, PASP, 99, 1214 
